
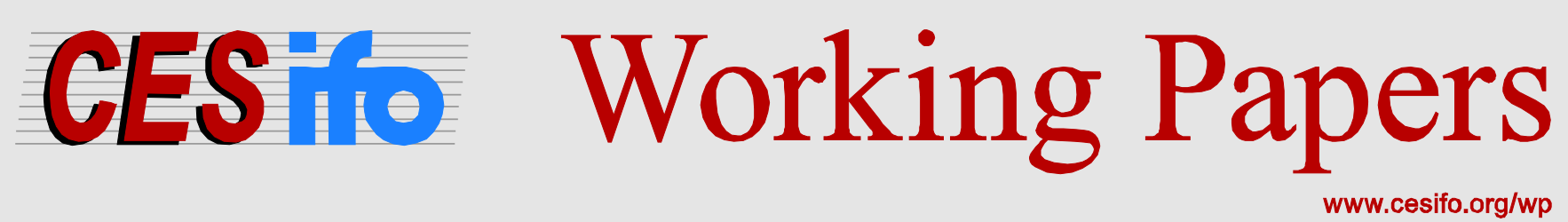

\title{
To Pool or not to Pool: Revisited
}

\author{
M. Hashem Pesaran \\ Qiankun Zhou
}

\author{
CESIFO WORKING PAPER NO. 5410 \\ CATEGORY 12: EMPIRICAL AND THEORETICAL METHODS \\ ORIGINAL VERSION: JUNE 2015 \\ THIS VERSION: DECEMBER 2016
}

An electronic version of the paper may be downloaded

- from the SSRN website: $\quad$ www.SSRN.com

- from the RePEc website: Www.RePEc.org

- from the CESifo website: www.CESifo-group.org/wp 


\title{
To Pool or not to Pool: Revisited
}

\begin{abstract}
This paper provides a new comparative analysis of pooled least squares and fixed effects estimators of the slope coefficients in the case of panel data models when the time dimension $(T)$ is fixed while the cross section dimension $(N)$ is allowed to increase without bounds. The individual effects are allowed to be correlated with the regressors, and the comparison is carried out in terms of an exponent coefficient, $\delta$, which measures the degree of pervasiveness of the fixed effects in the panel. The use of exponent $\delta$ allows us to distinguish between poolability of small $N$ dimensional panels with large $T$ from large $N$ dimensional panels with small $T$. It is shown that the pooled estimator remains consistent so long as $\delta<1$, and is asymptotically normally distributed if $\delta<1 / 2$, for a fixed $T$ and as $N \rightarrow \infty$. It is further shown that when $\delta<$ $1 / 2$, the pooled estimator is more efficient than the fixed effects estimator. We also propose a Hausman type diagnostic test of $\delta<1 / 2$ which could be used in practice as a simple test of poolability. In the case where $N$ and $T \rightarrow \infty$, such that $T=O\left(N^{\mathrm{d}}\right)$, for some $\mathrm{d}>0$, the condition for poolability generalizes to $\delta<(1-d) / 2$. Monte Carlo evidence supports the main theoretical findings and gives some indications of gains to be made from pooling when $\delta<(1-d) / 2$.
\end{abstract}

JEL-Code: C010, C230, C330.

Keywords: short panel, fixed effects estimator, pooled estimator, efficiency, diagnostic test.

\author{
M. Hashem Pesaran* \\ Department of Economics \\ University of Southern California \\ 3620 South Vermont Avenue \\ Kaprielian Hall 300 \\ USA - Los Angeles, CA 90089 \\ pesaran@usc.edu
}

\author{
Qiankun Zhou \\ Binghamton University \\ State University of New York \\ 4400 Vestal Parkway East \\ USA - Binghamton, NY 13902 \\ qzhou@binghamton.edu
}

*corresponding author

December 3, 2016

We would like to thank Ron Smith and Carlos Lamarche for helpful comments. 


\section{Introduction}

This paper re-examines the issue of pooling in standard panel data models with exogenous regressors in terms of an exponent coefficient, $0 \leq \delta \leq 1$, which measures the degree of pervasiveness of correlated individual effects, defined by

$$
\sum_{i=1}^{N} E\left|\eta_{i}\right|=O\left(N^{\delta}\right),
$$

where $N$ is the cross- section dimension of the panel, and $\eta_{i}$ is the mean zero random part of the individual effects. The use of exponent $\delta$ allows us to distinguish between poolability of small $N$ dimensional panels with large $T$ from large $N$ dimensional panels with small $T$. A set of coefficients could be heterogeneous for a finite $N$, nevertheless can be deemed as asymptotically homogeneous if their dispersion tends to zero as $N \rightarrow \infty$. We use this idea to motivate conditions under which pooling is valid in large $N$ dimensional panels, both when $T$ is fixed and when it rises with $N$.

Throughout we allow for non-zero correlations between the individual effects and the regressors, and as a result the pooled estimators will be biased in the standard case where $\delta=1$. We show that the choice between the pooled least squares (PLS) estimator and the fixed effects (FE) estimator depends on the value of $\delta$, with the PLS estimator being consistent for all values of $\delta$ except when $\delta=1$. For inference, the validity of the PLS estimator requires $\delta<1 / 2$. Both of these conditions are significantly weaker than the homogeneity assumption made in the literature requiring that $E\left|\eta_{i}\right|=0$ for all $i$. For example, when $\delta=0$ we could have a finite number of non-zero $E\left|\eta_{i}\right|$, or more generally when $E\left|\eta_{i}\right|=K \rho^{i}$, for a fixed positive constant $K$, and $0<\rho<1$. This corresponds to the sparsity assumption often made in the context of penalized regressions. But our analysis covers non-sparse structures by allowing the number of non-zero $E\left|\eta_{i}\right|$ 's to rise with $N$ but not proportionately. The degree to which the number of units with non-zero $E\left|\eta_{i}\right|$ is allowed to rise with $N$ is governed by $\delta$. For example, when $\delta=1 / 2$ the number of cross-section units with non-zero random effects could rise with $\sqrt{N}$, with the proportion of such units in total declining to zero at the rate of $N^{-1 / 2}$.

The exponent of pervasiveness of individual effects is also closely related to the exponent of cross-sectional dependence, $\alpha$, recently introduced in Bailey et al. (2016) to measure the degree of cross-sectional dependence in panels. Both exponents measure the degree of pervasiveness of heterogeneity, $\delta$ relates to the heterogeneity of the individual effects, and $\alpha$ the heterogeneity of factor loadings in a panel data model with a factor error structure. In a broad sense, $\delta$ can also be viewed as an exponent of cross-sectional dependence applied to the intercepts viewed as a common factor. 
Our analysis complements and provides further insights on the discussion of "pool or not to pool" in the panel literature. ${ }^{1}$ See for example, Baltagi et al (2000), and Baltagi (2008). More specifically, we derive the asymptotic properties of the pooled least squares estimator when $N$ is large and $T$ is fixed for different values of $\delta$, and derive the bias of PLS when $\delta=1$, and show that the pooled estimator is more efficient than the fixed effects estimator if $\delta<1 / 2$. We also establish the asymptotic equivalence of random effects and PLS estimators when $\delta<1$. In the case where $N$ and $T \rightarrow \infty$, such that $T=O\left(N^{d}\right)$, for some $d>0$, the condition for poolability generalizes to $\delta<(1-d) / 2$. Monte Carlo simulations are conducted to compare the finite sample properties of PLS and FE estimators. The results confirm the main theoretical findings and give some indication of the magnitudes of the gains involved from pooling when $\delta<(1-d) / 2$.

The analysis of this paper also shows the importance of knowing $\delta$ in the choice between PLS (or RE) and FE estimators. In the case of large $N$ and $T$ panels estimation of $\delta$ can be carried out using the approach of Bailey et al. (2016). But for short $T$ panels, which is of concern in this paper, such an approach will not be applicable and other suitable techniques will be required. Accordingly, we also propose a Hausman type diagnostic test of $\delta<(1-d) / 2$ which could be used in practice as a simple test of poolability of panel data models.

The rest of the paper is organized as follows. Section 2 sets out the model and its assumptions. Section 3 presents the main theoretical results on the consistency and asymptotic normality of PLS and FE estimators in terms of different values of $\delta$. The diagnostic test of poolability is presented in Section 4. Monte Carlo simulations are provided in Section 5, with some concluding remarks in Section 6.

\section{Panel data model}

Consider the standard panel data model

$$
\begin{aligned}
y_{i t} & =\alpha_{i}+\boldsymbol{\beta}^{\prime} \mathbf{x}_{i t}+u_{i t}, \text { for } i=1,2, \ldots, N ; t=1,2, \ldots, T \\
\alpha_{i} & =\alpha+\eta_{i} \text { for } i=1,2, \ldots, N
\end{aligned}
$$

where $\alpha_{i}$ are the individual effects, $\mathbf{x}_{i t}$ is a $k \times 1$ vector of regressors which we decompose as

$$
\mathbf{x}_{i t}=\eta_{i} \mathbf{g}_{t}+\mathbf{w}_{i t}, \text { for } i=1,2, \ldots, N ; t=1,2, \ldots, T .
$$

\footnotetext{
${ }^{1}$ There is also a related literature that considers the problem of pooling more generally and discusses the issue of pooling in the case of panel data models with heterogenous slopes. As a recent example, see Paap, Wang and Zhang (2015) and references cited therein. In this paper we focus on the issue of pooling in the context of standard panel data models with homogeneous slopes. But our approach and generalization of the concept of cross-sectional heterogeneity can also be applied to panel data models with heterogeneous slopes.
} 
$\eta_{i} \mathbf{g}_{t}$ represents the part of $\mathbf{x}_{i t}$ which is correlated with the individual effects, $\alpha_{i}$, with $\mathbf{g}_{t}$ being a $k \times 1$ vector of time effects, and $\mathbf{w}_{i t}$ is the part of $\mathbf{x}_{i t}$ which is distributed independently of the individual effects. This is a fairly general specification which allows for non-zero, possibly time-varying, correlations between $\mathbf{x}_{i t}$ and $\alpha_{i}$, and allows the regressors to have individualspecific effects and be cross-sectionally correlated. Additional individual-specific effects can be included in $\mathbf{x}_{i t}$ through $\mathbf{w}_{i t}$. For example, using (2.3), and assuming that $\overline{\mathbf{g}}=T^{-1} \sum_{t=1}^{T} \mathbf{g}_{t} \neq \mathbf{0}$, then

$$
\eta_{i}=\boldsymbol{\pi}^{\prime} \overline{\mathbf{x}}_{i}+v_{i}
$$

where ,

$$
\begin{aligned}
& \boldsymbol{\pi}^{\prime}=\left(\overline{\mathbf{g}}^{\prime} \overline{\mathbf{g}}\right)^{-1} \overline{\mathbf{g}}^{\prime}, v_{i}=-\left(\overline{\mathbf{g}}^{\prime} \overline{\mathbf{g}}\right)^{-1} \overline{\mathbf{g}}^{\prime} \overline{\mathbf{w}}_{i}, \\
& \overline{\mathbf{x}}_{i}=T^{-1} \sum_{t=1}^{T} \mathbf{x}_{i t}, \text { and } \overline{\mathbf{w}}_{i}=T^{-1} \sum_{t=1}^{T} \mathbf{w}_{i t},
\end{aligned}
$$

which is the same as Mundlak (1978) formulation of the individual effects in standard panel data models.

Throughout we assume $T$ is fixed and carry out our analysis for $N$ large. Except for the assumption regarding the individual effects, $\eta_{i}$, we make the following standard assumptions:

Assumption 1: The individual effects, $\eta_{i}$ for $i=1,2, \ldots, N$, are either deterministic and bounded (i.e. $\left|\eta_{i}\right|<K$ ), or stochastic with second order moments, $E\left(\eta_{i}^{2}\right)<K$, and distributed independently of $\mathbf{g}_{t}$ and $\mathbf{w}_{j t}$ for all $i, j$ and $t$; satisfying the conditions ${ }^{2}$

$$
N^{-1} \sum_{i=1}^{N} E\left|\eta_{i}\right|^{s}=O\left(N^{\delta-1}\right), \text { for } s=1 \text { and } 2, \text { where } 0 \leq \delta \leq 1 .
$$

Remark 2.1 The conditions of Assumption 1 are satisfied, for example, if there exists an ordering of the individual units such that for $\delta$ in the range $[0,1]$

$$
\begin{aligned}
\eta_{i} & =\varepsilon_{i}, \text { for } i=1,2, \ldots,\left[N^{\delta}\right], \\
& =0, \text { for } i=\left[N^{\delta}\right]+1,\left[N^{\delta}\right]+2, \ldots, N
\end{aligned}
$$

where $\left\{\varepsilon_{i}, i=1,2, \ldots, N\right\}$ is a sequence of random variables with zero means and finite variances such that

$$
\lim _{M \rightarrow \infty}\left(M^{-1} \sum_{i=1}^{M} E\left|\varepsilon_{i}\right|^{s}\right)=O(1), \text { for } s=1 \text { and } 2 .
$$

\footnotetext{
${ }^{2} K$ represents a generic finite positive constant.
} 
Then,

$$
N^{-1} \sum_{i=1}^{N} E\left|\eta_{i}\right|^{s}=N^{\delta-1}\left(N^{-\delta} \sum_{i=1}^{\left[N^{\delta}\right]}\left|\varepsilon_{i}\right|^{s}\right)=O\left(N^{\delta-1}\right) .
$$

Note that the above result holds even if $\varepsilon_{i}^{\prime} s$ are cross-sectionally correlated. Furthermore, the condition that $\eta_{i}=0$, for $i=\left[N^{\delta}\right]+1,\left[N^{\delta}\right]+2, \ldots, N$, can be relaxed by requiring. (See also Bailey et al. (2016)).

$$
\sum_{i=\left[N^{\delta}\right]+1}^{N} E\left|\eta_{i}\right|^{s}=O(1), \text { for } s=1 \text { and } 2 .
$$

This condition holds, for example, if $E\left|\eta_{i}\right|^{s}=\kappa_{i s} \rho_{s}^{i}$ for $i=\left[N^{\delta}\right]+1,\left[N^{\delta}\right]+2, \ldots, N$, where $\kappa_{\text {is }}$ are finite positive constants and $0 \leq \rho_{s}<1$.

Remark 2.2 Conditions (2.5) also imply

$$
N^{-1} \sum_{i=1}^{N} \eta_{i}^{2}=O_{p}\left(N^{\delta-1}\right) \text {, and } N^{-1} \sum_{i=1}^{N}\left|\eta_{i}\right|=O_{p}\left(N^{\delta-1}\right) \text {. }
$$

These results follow by application of Markov inequality to (2.5).

Assumption 2: (a) $u_{i t}$ is distributed independently of $\eta_{j}$ and $\mathbf{w}_{j t^{\prime}}$ for all $i, j, t$, and $t^{\prime}$. (b) $u_{i t} \sim \operatorname{IID}\left(0, \sigma_{u}^{2}\right), 0<\sigma_{u}^{2}<K$, and $E\left|u_{i t}\right|^{4+\epsilon}<K$, for some small positive $\epsilon$.

Assumption 3: The time effects, $\mathbf{g}_{t}$, are bounded such that $\left\|\mathbf{g}_{t} \mathbf{g}_{t}^{\prime}\right\|<K<\infty$, if $\mathbf{g}_{t}$ is deterministic and $E\left\|\mathbf{g}_{t} \mathbf{g}_{t}^{\prime}\right\|<K<\infty$, if $\mathbf{g}_{t}$ is stochastic. $\|\mathbf{A}\|$ represents the Frobenius norm of $\mathbf{A}$ defined by $\operatorname{Tr}\left(\mathbf{A} \mathbf{A}^{\prime}\right)^{1 / 2}$.

Assumption 4: The variables, $\mathbf{w}_{i t}$, are either deterministic and bounded, namely $\left\|\mathbf{w}_{i t}\right\|<$ $K<\infty$, or they satisfy the moment conditions $E\left\|\mathbf{w}_{i t}-\overline{\mathbf{w}}_{i}\right\|^{2}<K<\infty$, for all $i$ and $t$, where $\overline{\mathbf{w}}_{i}=T^{-1} \sum_{t=1}^{T} \mathbf{w}_{i t}$. Similarly, $E\left\|\overline{\mathbf{w}}_{i}-\overline{\mathbf{w}}\right\|^{2}<K<\infty$, for all $i$, where $\overline{\mathbf{w}}=N^{-1} \sum_{i=1}^{N} \overline{\mathbf{w}}_{i}$.

Assumption 5: The $k \times k$ matrices

$$
\begin{aligned}
\boldsymbol{\Omega}_{P, N} & =\frac{1}{N T} \sum_{i=1}^{N} \sum_{t=1}^{T}\left(\mathbf{w}_{i t}-\overline{\mathbf{w}}\right)\left(\mathbf{w}_{i t}-\overline{\mathbf{w}}\right)^{\prime}, \\
\boldsymbol{\Omega}_{F E, N} & =\frac{1}{N T} \sum_{i=1}^{N} \sum_{t=1}^{T}\left(\mathbf{w}_{i t}-\overline{\mathbf{w}}_{i}\right)\left(\mathbf{w}_{i t}-\overline{\mathbf{w}}_{i}\right)^{\prime},
\end{aligned}
$$

are positive definite for all $N$, and as $N \rightarrow \infty$. The probability limits of $\boldsymbol{\Omega}_{P, N}$ and $\boldsymbol{\Omega}_{F E, N}$, as $N$ tends to infinity, will be denoted by $\boldsymbol{\Omega}_{P}$ and $\boldsymbol{\Omega}_{F E}$, respectively.

Remark 2.3 Note that $E\left\|\mathbf{w}_{i t}-\overline{\mathbf{w}}_{i}\right\| \leq\left[E\left\|\overline{\mathbf{w}}_{i}-\overline{\mathbf{w}}\right\|^{2}\right]^{1 / 2}<K<\infty$, and $E\left\|\overline{\mathbf{w}}_{i}-\overline{\mathbf{w}}\right\| \leq$ $\left[E\left\|\overline{\mathbf{w}}_{i}-\overline{\mathbf{w}}\right\|^{2}\right]^{1 / 2}<K<\infty$. Hence under Assumption 4 we also have $E\left\|\left(\mathbf{w}_{i t}-\overline{\mathbf{w}}\right)\right\|=E\left\|\left(\mathbf{w}_{i t}-\overline{\mathbf{w}}_{i}+\overline{\mathbf{w}}_{i}-\overline{\mathbf{w}}\right)\right\| \leq E\left\|\left(\mathbf{w}_{i t}-\overline{\mathbf{w}}_{i}\right)\right\|+E\left\|\left(\overline{\mathbf{w}}_{i}-\overline{\mathbf{w}}\right)\right\|<K<\infty$. 


\section{Pooled least squares and FE estimators}

The PLS and FE estimators, $\hat{\boldsymbol{\beta}}_{P}$ and $\hat{\boldsymbol{\beta}}_{F E}$, respectively, can be written as

$$
\hat{\boldsymbol{\beta}}_{P}=\mathbf{Q}_{P, N}^{-1} \mathbf{q}_{P, N},
$$

and

$$
\hat{\boldsymbol{\beta}}_{F E}=\mathbf{Q}_{F E, N}^{-1} \mathbf{q}_{F E, N},
$$

where

$$
\begin{gathered}
\mathbf{Q}_{P, N}=\frac{1}{N T} \sum_{i=1}^{N} \sum_{t=1}^{T}\left(\mathbf{x}_{i t}-\overline{\mathbf{x}}\right)\left(\mathbf{x}_{i t}-\overline{\mathbf{x}}\right)^{\prime}, \mathbf{q}_{P, N}=\frac{1}{N T} \sum_{i=1}^{N} \sum_{t=1}^{T}\left(\mathbf{x}_{i t}-\overline{\mathbf{x}}\right)\left(y_{i t}-\bar{y}\right), \\
\mathbf{Q}_{F E, N}=\frac{1}{N T} \sum_{i=1}^{N} \sum_{t=1}^{T}\left(\mathbf{x}_{i t}-\overline{\mathbf{x}}_{i}\right)\left(\mathbf{x}_{i t}-\overline{\mathbf{x}}_{i}\right)^{\prime}, \mathbf{q}_{F E, N}=\frac{1}{N T} \sum_{i=1}^{N} \sum_{t=1}^{T}\left(\mathbf{x}_{i t}-\overline{\mathbf{x}}_{i}\right)\left(y_{i t}-\bar{y}_{i}\right),
\end{gathered}
$$

and

$$
\begin{aligned}
\overline{\mathbf{x}} & =(N T)^{-1} \sum_{i=1}^{N} \sum_{t=1}^{T} \mathbf{x}_{i t}, \bar{y}=(N T)^{-1} \sum_{i=1}^{N} \sum_{t=1}^{T} y_{i t}, \\
\overline{\mathbf{x}}_{i} & =T^{-1} \sum_{t=1}^{T} \mathbf{x}_{i t}, \bar{y}_{i}=T^{-1} \sum_{t=1}^{T} y_{i t} .
\end{aligned}
$$

To derive the properties of these estimators, using (2.3), we first note that

$$
\mathbf{x}_{i t}-\overline{\mathbf{x}}=\left(\eta_{i} \mathbf{g}_{t}-\bar{\eta} \overline{\mathbf{g}}\right)+\left(\mathbf{w}_{i t}-\overline{\mathbf{w}}\right)
$$

and

$$
y_{i t}-\bar{y}=\eta_{i}-\bar{\eta}+\boldsymbol{\beta}^{\prime}\left(\mathbf{x}_{i t}-\overline{\mathbf{x}}\right)+\left(u_{i t}-\bar{u}\right),
$$

where

$$
\overline{\mathbf{g}}=T^{-1} \sum_{t=1}^{T} \mathbf{g}_{t}, \bar{\eta}=N^{-1} \sum_{i=1}^{N} \eta_{i}, \text { and } \bar{u}=N^{-1} \sum_{i=1}^{N} u_{i}
$$

\subsection{The PLS estimator}

Starting with the PLS estimator, using (3.7) in (3.3) we have

$$
\begin{aligned}
\mathbf{Q}_{P, N}= & \frac{1}{N T} \sum_{i=1}^{N} \sum_{t=1}^{T}\left(\eta_{i} \mathbf{g}_{t}+\mathbf{w}_{i t}-\bar{\eta} \overline{\mathbf{g}}-\overline{\mathbf{w}}\right)\left(\eta_{i} \mathbf{g}_{t}+\mathbf{w}_{i t}-\bar{\eta} \overline{\mathbf{g}}-\overline{\mathbf{w}}\right)^{\prime} \\
= & \frac{1}{N T} \sum_{i=1}^{N} \sum_{t=1}^{T}\left(\mathbf{w}_{i t}-\overline{\mathbf{w}}\right)\left(\mathbf{w}_{i t}-\overline{\mathbf{w}}\right)^{\prime}+\left(N^{-1} \sum_{i=1}^{N} \eta_{i}^{2}\right)\left(T^{-1} \sum_{t=1}^{T} \mathbf{g}_{t} \mathbf{g}_{t}^{\prime}\right)-\bar{\eta}^{2}\left(\overline{\mathbf{g}} \overline{\mathbf{g}}^{\prime}\right) \\
& +\frac{1}{N T} \sum_{i=1}^{N} \sum_{t=1}^{T} \eta_{i}\left[\left(\mathbf{w}_{i t}-\overline{\mathbf{w}}\right) \mathbf{g}_{t}^{\prime}+\mathbf{g}_{t}\left(\mathbf{w}_{i t}-\overline{\mathbf{w}}\right)^{\prime}\right]
\end{aligned}
$$


Similarly, using (3.8) in (3.3) we have

$$
\begin{aligned}
\mathbf{q}_{P, N} & =\frac{1}{N T} \sum_{i=1}^{N} \sum_{t=1}^{T}\left(\mathbf{x}_{i t}-\overline{\mathbf{x}}\right)\left(y_{i t}-\bar{y}\right) \\
& =\frac{1}{N T} \sum_{i=1}^{N} \sum_{t=1}^{T}\left(\mathbf{x}_{i t}-\overline{\mathbf{x}}\right)\left[\eta_{i}-\bar{\eta}+\boldsymbol{\beta}^{\prime}\left(\mathbf{x}_{i t}-\overline{\mathbf{x}}\right)+u_{i t}-\bar{u}\right] \\
& =\mathbf{Q}_{P, N} \boldsymbol{\beta}+\frac{1}{N T} \sum_{i=1}^{N} \sum_{t=1}^{T}\left(\mathbf{x}_{i t}-\overline{\mathbf{x}}\right)\left(\eta_{i}-\bar{\eta}+u_{i t}-\bar{u}\right),
\end{aligned}
$$

which upon using (3.7) can be written as

$$
\mathbf{q}_{P, N}=\mathbf{Q}_{P, N} \boldsymbol{\beta}+\frac{1}{N T} \sum_{i=1}^{N} \sum_{t=1}^{T}\left(\mathbf{w}_{i t}-\overline{\mathbf{w}}\right)\left(\eta_{i}+u_{i t}\right)+\frac{1}{N T} \sum_{i=1}^{N} \sum_{t=1}^{T}\left(\eta_{i} \mathbf{g}_{t}-\bar{\eta} \overline{\mathbf{g}}\right)\left(\eta_{i}+u_{i t}\right),
$$

which in turn yields

$$
\hat{\boldsymbol{\beta}}_{P}=\boldsymbol{\beta}+\mathbf{Q}_{P, N}^{-1}\left[\frac{1}{N T} \sum_{i=1}^{N} \sum_{t=1}^{T}\left(\mathbf{w}_{i t}-\overline{\mathbf{w}}\right)\left(\eta_{i}+u_{i t}\right)+\frac{1}{N T} \sum_{i=1}^{N} \sum_{t=1}^{T}\left(\eta_{i} \mathbf{g}_{t}-\bar{\eta} \overline{\mathbf{g}}\right)\left(\eta_{i}+u_{i t}\right)\right] .
$$

Furthermore,

$$
\begin{aligned}
\frac{1}{N T} \sum_{i=1}^{N} \sum_{t=1}^{T}\left(\eta_{i} \mathbf{g}_{t}-\bar{\eta} \overline{\mathbf{g}}\right)\left(\eta_{i}+u_{i t}\right) & =\frac{1}{N T} \sum_{i=1}^{N} \sum_{t=1}^{T}\left(\eta_{i}^{2} \mathbf{g}_{t}-\bar{\eta} \eta_{i} \overline{\mathbf{g}}\right)+\frac{1}{N T} \sum_{i=1}^{N} \sum_{t=1}^{T}\left(\eta_{i} \mathbf{g}_{t}-\bar{\eta} \overline{\mathbf{g}}\right) u_{i t} \\
& =\frac{1}{N} \sum_{i=1}^{N}\left(\eta_{i}^{2}-\bar{\eta} \eta_{i}\right) \overline{\mathbf{g}}+\frac{1}{N T} \sum_{i=1}^{N} \sum_{t=1}^{T}\left(\eta_{i} \mathbf{g}_{t}-\bar{\eta} \overline{\mathbf{g}}\right) u_{i t} \\
& =\left[N^{-1} \sum_{i=1}^{N}\left(\eta_{i}-\bar{\eta}\right)^{2}\right] \overline{\mathbf{g}}+\frac{1}{N T} \sum_{i=1}^{N} \sum_{t=1}^{T} \eta_{i} \mathbf{g}_{t} u_{i t}-\bar{\eta} \overline{\mathbf{g}} \bar{u}(.3 .12)
\end{aligned}
$$

But under Assumption 1 we have

$$
N^{-1} \sum_{i=1}^{N} E\left|\eta_{i}\right|^{2}=O\left(N^{\delta-1}\right) \text {, and } E|\bar{\eta}| \leq N^{-1} \sum_{i=1}^{N}\left|\eta_{i}\right|=O\left(N^{\delta-1}\right),
$$

and since $\eta_{i}$ is distributed independently of $\mathbf{g}$ and $u_{i t}$, then

$$
\begin{aligned}
E\left|\frac{1}{N T} \sum_{i=1}^{N} \sum_{t=1}^{T}\left(\eta_{i} \mathbf{g}_{t}-\bar{\eta} \overline{\mathbf{g}}\right)\left(\eta_{i}+u_{i t}\right)\right| \leq & {\left[N^{-1} \sum_{i=1}^{N} E\left(\eta_{i}-\bar{\eta}\right)^{2}\right] E(\||\overline{\mathbf{g}}|\|) } \\
& +\sup _{i, t} E\left|u_{i t}\right| \sup _{t} E\left(\left\|\mathbf{g}_{t}\right\|\right)\left(N^{-1} \sum_{i=1}^{N} E\left|\eta_{i}\right|\right)+E|\bar{\eta}| E|\bar{u}| E(\||\overline{\mathbf{g}}|\|) \\
= & O\left(N^{\delta-1}\right)
\end{aligned}
$$


Similarly

$$
\begin{aligned}
\left\|\frac{1}{N T} \sum_{i=1}^{N} \sum_{t=1}^{T} \eta_{i}\left[\left(\mathbf{w}_{i t}-\overline{\mathbf{w}}\right) \mathbf{g}_{t}^{\prime}+\mathbf{g}_{t}\left(\mathbf{w}_{i t}-\overline{\mathbf{w}}\right)^{\prime}\right]\right\| & \leq \frac{2}{N T} \sum_{i=1}^{N} \sum_{t=1}^{T}\left|\eta_{i}\right|\left\|\left(\mathbf{w}_{i t}-\overline{\mathbf{w}}\right)\right\|\left\|\mathbf{g}_{t}\right\| \\
& =\frac{2}{N} \sum_{i=1}^{N}\left|\eta_{i}\right|\left[T^{-1} \sum_{t=1}^{T}\left\|\left(\mathbf{w}_{i t}-\overline{\mathbf{w}}\right)\right\|\left\|\mathbf{g}_{t}\right\|\right]
\end{aligned}
$$

and since under Assumption $1, \eta_{i}$ is distributed independently of $\mathbf{g}_{t}$ and $\mathbf{w}_{i t}$, we have

$E\left\|\frac{1}{N T} \sum_{i=1}^{N} \sum_{t=1}^{T} \eta_{i}\left[\left(\mathbf{w}_{i t}-\overline{\mathbf{w}}\right) \mathbf{g}_{t}^{\prime}+\mathbf{g}_{t}\left(\mathbf{w}_{i t}-\overline{\mathbf{w}}\right)^{\prime}\right]\right\| \leq \frac{2}{N} \sum_{i=1}^{N} E\left|\eta_{i}\right|\left[\left\{T^{-1} \sum_{t=1}^{T} E\left[\left\|\left(\mathbf{w}_{i t}-\overline{\mathbf{w}}\right)\right\|\left\|\mathbf{g}_{t}\right\|\right]\right\}\right]$.

However, by Cauchy-Schwarz inequality and under Assumptions 3 and 4

$$
E\left[\left\|\left(\mathbf{w}_{i t}-\overline{\mathbf{w}}\right)\right\|\left\|\mathbf{g}_{t}\right\|\right] \leq\left[E\left\|\left(\mathbf{w}_{i t}-\overline{\mathbf{w}}\right)\right\|^{2}\right]^{1 / 2}\left[E\left\|\mathbf{g}_{t}\right\|^{2}\right]^{1 / 2}<K,
$$

and

$$
E\left\|\frac{1}{N T} \sum_{i=1}^{N} \sum_{t=1}^{T} \eta_{i}\left[\left(\mathbf{w}_{i t}-\overline{\mathbf{w}}\right) \mathbf{g}_{t}^{\prime}+\mathbf{g}_{t}\left(\mathbf{w}_{i t}-\overline{\mathbf{w}}\right)^{\prime}\right]\right\| \leq \frac{2 K}{N} \sum_{i=1}^{N} E\left|\eta_{i}\right|=O\left(N^{\delta-1}\right) .
$$

Using (3.13) and the above result in (3.9) we obtain

$$
\mathbf{Q}_{P, N}=\frac{1}{N T} \sum_{i=1}^{N} \sum_{t=1}^{T}\left(\mathbf{w}_{i t}-\overline{\mathbf{w}}\right)\left(\mathbf{w}_{i t}-\overline{\mathbf{w}}\right)^{\prime}+O_{p}\left(N^{\delta-1}\right)
$$

which establishes that under $\delta<1$ (for a fixed $T$ and as $N \rightarrow \infty$ )

$$
\mathbf{Q}_{P, N} \rightarrow p \boldsymbol{\Omega}_{P}=\lim _{N \rightarrow \infty} \frac{1}{N T} \sum_{i=1}^{N} \sum_{t=1}^{T} E\left[\left(\mathbf{w}_{i t}-\overline{\mathbf{w}}\right)\left(\mathbf{w}_{i t}-\overline{\mathbf{w}}\right)^{\prime}\right]>0 .
$$

Consider now the second component of (3.11), and note from (3.12) that since by assumption $\eta_{i}, u_{i t}$, and $\mathbf{g}_{t}$ are distributed independently, then

$$
\begin{aligned}
E\left\|\frac{1}{N T} \sum_{i=1}^{N} \sum_{t=1}^{T} \eta_{i} \mathbf{g}_{t} u_{i t}\right\| & \leq \frac{1}{N T} \sum_{i=1}^{N} \sum_{t=1}^{T} E\left|\eta_{i}\right| E\left\|\mathbf{g}_{t}\right\| E\left|u_{i t}\right| \\
& \leq \frac{K}{N} \sum_{i=1}^{N} E\left|\eta_{i}\right|=O\left(N^{\delta-1}\right) .
\end{aligned}
$$

Hence, in view of (3.14) and using the above results we have

$$
\hat{\boldsymbol{\beta}}_{P}=\boldsymbol{\beta}+\boldsymbol{\Omega}_{P}^{-1}\left[\frac{1}{N T} \sum_{i=1}^{N} \sum_{t=1}^{T}\left(\mathbf{w}_{i t}-\overline{\mathbf{w}}\right)\left(\eta_{i}+u_{i t}\right)\right]+O_{p}\left(N^{\delta-1}\right) .
$$


Furthermore

$$
\frac{1}{N T} \sum_{i=1}^{N} \sum_{t=1}^{T}\left(\mathbf{w}_{i t}-\overline{\mathbf{w}}\right) \eta_{i}=\frac{1}{N} \sum_{i=1}^{N}\left(\overline{\mathbf{w}}_{i}-\overline{\mathbf{w}}\right) \eta_{i}
$$

and since by Assumption $1, \eta_{i}$ and $\overline{\mathbf{w}}_{i}-\overline{\mathbf{w}}$ are independently distributed and by Assumption $4, E\left\|\overline{\mathbf{w}}_{i}-\overline{\mathbf{w}}\right\|<K$, then

$$
E\left\|\frac{1}{N T} \sum_{i=1}^{N} \sum_{t=1}^{T}\left(\mathbf{w}_{i t}-\overline{\mathbf{w}}\right) \eta_{i}\right\| \leq \frac{1}{N} \sum_{i=1}^{N} E\left|\eta_{i}\right| E\left\|\overline{\mathbf{w}}_{i}-\overline{\mathbf{w}}\right\| \leq \frac{K}{N} \sum_{i=1}^{N} E\left|\eta_{i}\right|=O\left(N^{\delta-1}\right) .
$$

Therefore, (3.15) simplifies further to

$$
\hat{\boldsymbol{\beta}}_{P}=\boldsymbol{\beta}+\boldsymbol{\Omega}_{P}^{-1}\left[\frac{1}{N T} \sum_{i=1}^{N} \sum_{t=1}^{T}\left(\mathbf{w}_{i t}-\overline{\mathbf{w}}\right) u_{i t}\right]+O_{p}\left(N^{\delta-1}\right) .
$$

Using this result and noting that under Assumptions 2 and 4,

$$
\frac{1}{N T} \sum_{i=1}^{N} \sum_{t=1}^{T}\left(\mathbf{w}_{i t}-\overline{\mathbf{w}}\right) u_{i t} \rightarrow_{p} \mathbf{0},
$$

we have the following proposition.

Proposition 3.1 Consider the panel data model given by equations (2.1), (2.2) and (2.3) and suppose that Assumptions 1-5 hold. Then the pooled least square estimator defined by (3.1) is consistent for estimation of $\beta$, as long as $\delta<1$.

Remark 3.2 The bias of the pooled least squares estimator in the case of $\delta=1$ is given by

$$
p \lim _{N \rightarrow \infty}\left(\hat{\boldsymbol{\beta}}_{P}\right)=\boldsymbol{\beta}+\sigma_{\eta}^{2} \mathbf{Q}_{P}^{-1} \overline{\mathbf{g}}
$$

where

$$
\sigma_{\eta}^{2}=\lim _{N \rightarrow \infty} N^{-1} \sum_{i=1}^{N}\left(\eta_{i}-\bar{\eta}\right)^{2}
$$

and

$$
\mathbf{Q}_{P}=\lim _{N \rightarrow \infty} \frac{1}{N T} \sum_{i=1}^{N} \sum_{t=1}^{T} E\left[\left(\mathbf{x}_{i t}-\overline{\mathbf{x}}\right)\left(\mathbf{x}_{i t}-\overline{\mathbf{x}}\right)^{\prime}\right] .
$$

For a derivation see Section 26.3 in Pesaran (2015). As a corollary it also follows that Hausman's (1978) mis-specification test that compares the pooled and FE estimators will only be consistent if $\delta<1$. 
To derive the asymptotic distribution of $\hat{\boldsymbol{\beta}}_{P}$ we note that

$$
\sqrt{N}\left(\hat{\boldsymbol{\beta}}_{P}-\boldsymbol{\beta}\right)=\boldsymbol{\Omega}_{P}^{-1}\left[\frac{1}{T \sqrt{N}} \sum_{i=1}^{N} \sum_{t=1}^{T}\left(\mathbf{w}_{i t}-\overline{\mathbf{w}}\right) u_{i t}\right]+O_{p}\left(N^{\delta-1 / 2}\right) .
$$

Also under Assumptions 2, 4 and 5, using standard results from panel data literature, we have (for a fixed $T$ and as $N \rightarrow \infty$ )

$$
\frac{1}{T \sqrt{N}} \sum_{i=1}^{N} \sum_{t=1}^{T}\left(\mathbf{w}_{i t}-\overline{\mathbf{w}}\right) u_{i t} \rightarrow{ }_{d} N\left(\mathbf{0}, \sigma_{u}^{2} T^{-1} \boldsymbol{\Omega}_{P}\right) .
$$

Hence, for a fixed $T$ and as $N \rightarrow \infty$

$$
\sqrt{N}\left(\hat{\boldsymbol{\beta}}_{P}-\boldsymbol{\beta}\right) \rightarrow_{d} N\left(\mathbf{0}, \sigma_{u}^{2} T^{-1} \boldsymbol{\Omega}_{P}^{-1}\right), \text { if } \delta<1 / 2
$$

\subsection{The FE estimator}

Consider now the FE estimator, $\hat{\boldsymbol{\beta}}_{F E}$, defined by (3.2). Then using (3.4) we obtain

$$
\sqrt{N}\left(\hat{\boldsymbol{\beta}}_{F E}-\boldsymbol{\beta}\right)=\mathbf{Q}_{F E, N}^{-1}\left[\frac{1}{T \sqrt{N}} \sum_{i=1}^{N} \sum_{t=1}^{T}\left(\mathbf{x}_{i t}-\overline{\mathbf{x}}_{i}\right)\left(u_{i t}-\bar{u}_{i}\right)\right] .
$$

Noting that $\mathbf{x}_{i t}-\overline{\mathbf{x}}_{i}=\left(\mathbf{w}_{i t}-\overline{\mathbf{w}}_{i}\right)+\eta_{i}\left(\mathbf{g}_{t}-\overline{\mathbf{g}}\right)$, and $y_{i t}-\bar{y}_{i}=\boldsymbol{\beta}^{\prime}\left(\mathbf{x}_{i t}-\overline{\mathbf{x}}_{i}\right)+\left(u_{i t}-\bar{u}_{i}\right)$, we also have

$$
\begin{aligned}
\mathbf{Q}_{F E, N}= & \frac{1}{N T} \sum_{i=1}^{N} \sum_{t=1}^{T}\left(\mathbf{x}_{i t}-\overline{\mathbf{x}}_{i}\right)\left(\mathbf{x}_{i t}-\overline{\mathbf{x}}_{i}\right)^{\prime} \\
= & \frac{1}{N T} \sum_{i=1}^{N} \sum_{t=1}^{T}\left(\mathbf{w}_{i t}-\overline{\mathbf{w}}_{i}\right)\left(\mathbf{w}_{i t}-\overline{\mathbf{w}}_{i}\right)^{\prime}+\frac{1}{N T} \sum_{i=1}^{N} \sum_{t=1}^{T} \eta_{i}\left(\mathbf{w}_{i t}-\overline{\mathbf{w}}_{i}\right)\left(\mathbf{g}_{t}-\overline{\mathbf{g}}\right)^{\prime} \\
& +\frac{1}{N T} \sum_{i=1}^{N} \sum_{t=1}^{T} \eta_{i}\left(\mathbf{g}_{t}-\overline{\mathbf{g}}\right)\left(\mathbf{w}_{i t}-\overline{\mathbf{w}}_{i}\right)^{\prime}+\left(\frac{1}{N} \sum_{i=1}^{N} \eta_{i}^{2}\right)\left(\frac{1}{T} \sum_{t=1}^{T}\left(\mathbf{g}_{t}-\overline{\mathbf{g}}\right)\left(\mathbf{g}_{t}-\overline{\mathbf{g}}\right)^{\prime}\right),
\end{aligned}
$$

and

$$
\begin{aligned}
\frac{1}{T \sqrt{N}} \sum_{i=1}^{N} \sum_{t=1}^{T}\left(\mathbf{x}_{i t}-\overline{\mathbf{x}}_{i}\right)\left(u_{i t}-\bar{u}_{i}\right)= & \frac{1}{T \sqrt{N}} \sum_{i=1}^{N} \sum_{t=1}^{T}\left[\left(\mathbf{w}_{i t}-\overline{\mathbf{w}}_{i}\right)+\eta_{i}\left(\mathbf{g}_{t}-\overline{\mathbf{g}}\right)\right]\left(u_{i t}-\bar{u}_{i}\right) \\
= & \frac{1}{T \sqrt{N}} \sum_{i=1}^{N} \sum_{t=1}^{T}\left(u_{i t}-\bar{u}_{i}\right)\left(\mathbf{w}_{i t}-\overline{\mathbf{w}}_{i}\right) \\
& +\frac{1}{T \sqrt{N}} \sum_{i=1}^{N} \sum_{t=1}^{T} \eta_{i}\left(u_{i t}-\bar{u}_{i}\right)\left(\mathbf{g}_{t}-\overline{\mathbf{g}}\right) .
\end{aligned}
$$


Under Assumptions 1-4, using the above results and following the same line of reasoning as in Section 3.1 we have (for a fixed $T$ and as $N \rightarrow \infty$ )

$$
\begin{aligned}
\mathbf{Q}_{F E, N}= & \lim _{N \rightarrow \infty} \frac{1}{N T} \sum_{i=1}^{N} \sum_{t=1}^{T} E\left[\left(\mathbf{w}_{i t}-\overline{\mathbf{w}}_{i}\right)\left(\mathbf{w}_{i t}-\overline{\mathbf{w}}_{i}\right)^{\prime}\right] \\
& +\left(\frac{1}{N} \sum_{i=1}^{N} E\left(\eta_{i}^{2}\right)\right)\left(\frac{1}{T} \sum_{t=1}^{T} E\left[\left(\mathbf{g}_{t}-\overline{\mathbf{g}}\right)\left(\mathbf{g}_{t}-\overline{\mathbf{g}}\right)^{\prime}\right]\right) \\
= & \boldsymbol{\Omega}_{F E}+O_{p}\left(N^{\delta-1}\right),
\end{aligned}
$$

where

$$
\boldsymbol{\Omega}_{F E}=\lim _{N \rightarrow \infty} \frac{1}{N T} \sum_{i=1}^{N} \sum_{t=1}^{T} E\left[\left(\mathbf{w}_{i t}-\overline{\mathbf{w}}_{i}\right)\left(\mathbf{w}_{i t}-\overline{\mathbf{w}}_{i}\right)^{\prime}\right] .
$$

Similarly, since $\eta_{i}$ is distributed independently of $u_{i t}$ and $\mathbf{g}_{t}$, then

$$
\begin{aligned}
E\left|\frac{1}{\sqrt{N} T} \sum_{i=1}^{N} \sum_{t=1}^{T} \eta_{i}\left(u_{i t}-\bar{u}_{i}\right)\left(\mathbf{g}_{t}-\overline{\mathbf{g}}\right)\right| & \leq \frac{1}{\sqrt{N} T}\left|\sum_{i=1}^{N} \sum_{t=1}^{T} E\right| \eta_{i}|E|\left(u_{i t}-\bar{u}_{i}\right)\left(\mathbf{g}_{t}-\overline{\mathbf{g}}\right)|| \\
& \leq \sup _{i} E\left|\left(u_{i t}-\bar{u}_{i}\right)\left(\mathbf{g}_{t}-\overline{\mathbf{g}}\right)\right|\left(N^{-1 / 2} \sum_{i=1}^{N} E\left|\eta_{i}\right|\right) .
\end{aligned}
$$

But $E\left|\left(u_{i t}-\bar{u}_{i}\right)\left(\mathbf{g}_{t}-\overline{\mathbf{g}}\right)\right| \leq\left[E\left(u_{i t}-\bar{u}_{i}\right)^{2}\right]^{1 / 2}\left[E\left\|\mathbf{g}_{t}-\overline{\mathbf{g}}\right\|^{2}\right]^{1 / 2}<K$, and by Assumptions 1 and 2 , it follows that

$$
E\left|\frac{1}{\sqrt{N} T} \sum_{i=1}^{N} \sum_{t=1}^{T} \eta_{i}\left(u_{i t}-\bar{u}_{i}\right)\left(\mathbf{g}_{t}-\overline{\mathbf{g}}\right)\right| \leq O\left(N^{\delta-1 / 2}\right) .
$$

Finally, under Assumptions 2-4, using standard results from panel data literature we have

$$
\frac{1}{T \sqrt{N}} \sum_{i=1}^{N} \sum_{t=1}^{T}\left(u_{i t}-\bar{u}_{i}\right)\left(\mathbf{w}_{i t}-\overline{\mathbf{w}}_{i}\right) \rightarrow_{d} N\left(\mathbf{0}, \sigma_{u}^{2} T^{-1} \boldsymbol{\Omega}_{F E}\right),
$$

where $\boldsymbol{\Omega}_{F E}$ is already defined by (3.21).

Consequently, combining the above derivation yields

$$
\sqrt{N}\left(\hat{\boldsymbol{\beta}}_{F E}-\boldsymbol{\beta}\right)=\mathbf{Q}_{F E, N}^{-1}\left[\frac{1}{T \sqrt{N}} \sum_{i=1}^{N} \sum_{t=1}^{T}\left(\mathbf{w}_{i t}-\overline{\mathbf{w}}_{i}\right)\left(u_{i t}-\bar{u}_{i}\right)\right]+O\left(N^{\delta-1 / 2}\right) .
$$

Therefore, for a fixed $T$ and as $N \rightarrow \infty$, we have

$$
\sqrt{N}\left(\hat{\boldsymbol{\beta}}_{F E}-\boldsymbol{\beta}\right) \rightarrow_{d} N\left(\mathbf{0}, \sigma_{u}^{2} T^{-1} \boldsymbol{\Omega}_{F E}^{-1}\right), \text { for } \delta<1 / 2 .
$$

Using (3.18) and the above result now yields the following proposition 
Proposition 3.3 Suppose that the exponent coefficient, $\delta$, defined by Assumption 1, is less than $1 / 2$, and Assumptions 1-5 hold. Then for a fixed $T$, and as $N \rightarrow \infty$

$$
\sqrt{N T}\left(\hat{\boldsymbol{\beta}}_{P}-\boldsymbol{\beta}\right) \rightarrow_{d} N\left(\mathbf{0}, \sigma_{u}^{2} \boldsymbol{\Omega}_{P}^{-1}\right)
$$

and

$$
\sqrt{N T}\left(\hat{\boldsymbol{\beta}}_{F E}-\boldsymbol{\beta}\right) \rightarrow_{d} N\left(\mathbf{0}, \sigma_{u}^{2} \boldsymbol{\Omega}_{F E}^{-1}\right) .
$$

Furthermore, $\hat{\boldsymbol{\beta}}_{P}$ is asymptotically more efficient than $\hat{\boldsymbol{\beta}}_{F E}$, as long as $\delta<1 / 2$.

Remark 3.4 In the case where $T=O\left(N^{d}\right)$, for some $d>0$, the condition for $\hat{\boldsymbol{\beta}}_{P}$ to be asymptotically more efficient than $\hat{\boldsymbol{\beta}}_{F E}$ is given by $\delta<(1-d) / 2$, as $N$ and $T \rightarrow \infty$. This result follows if the expressions in (3.17) and (3.22) are pre-multiplied by $\sqrt{T}$, and $T$ replaced by $\left(N^{d}\right)$. When $N$ and $T$ expand at the same rate, and $d=1$, the FE estimator is always more efficient.

To establish the relative asymptotic efficiency of $\hat{\boldsymbol{\beta}}_{P}$ we first note that

$$
\left[\operatorname{Asy} \operatorname{Var}\left(\sqrt{T N} \hat{\boldsymbol{\beta}}_{P}\right)\right]^{-1}-\left[\operatorname{Asy} \operatorname{Var}\left(\sqrt{T N} \hat{\boldsymbol{\beta}}_{F E}\right)\right]^{-1}=\sigma_{u}^{-2}\left[\boldsymbol{\Omega}_{P}-\boldsymbol{\Omega}_{F E}\right] .
$$

Also, we note that since

$\frac{1}{N T} \sum_{i=1}^{N} \sum_{t=1}^{T}\left(\mathbf{w}_{i t}-\overline{\mathbf{w}}\right)\left(\mathbf{w}_{i t}-\overline{\mathbf{w}}\right)^{\prime}=\frac{1}{N T} \sum_{i=1}^{N} \sum_{t=1}^{T}\left(\mathbf{w}_{i t}-\overline{\mathbf{w}}_{i}\right)\left(\mathbf{w}_{i t}-\overline{\mathbf{w}}_{i}\right)^{\prime}+\frac{1}{N} \sum_{i=1}^{N}\left(\overline{\mathbf{w}}_{i}-\overline{\mathbf{w}}\right)\left(\overline{\mathbf{w}}_{i}-\overline{\mathbf{w}}\right)^{\prime}$,

then

$$
\boldsymbol{\Omega}_{P}=\boldsymbol{\Omega}_{F E}+\boldsymbol{\Omega}_{C}
$$

where

$$
\boldsymbol{\Omega}_{C}=\lim _{N \rightarrow \infty} \frac{1}{N} \sum_{i=1}^{N} E\left[\left(\overline{\mathbf{w}}_{i}-\overline{\mathbf{w}}\right)\left(\overline{\mathbf{w}}_{i}-\overline{\mathbf{w}}\right)^{\prime}\right]
$$

and by Assumption 5, $\boldsymbol{\Omega}_{C}$ is a positive definite matrix. Using (3.25) in (3.24) we have

$$
\left[\operatorname{Asy} \operatorname{Var}\left(\sqrt{T N} \hat{\boldsymbol{\beta}}_{P}\right)\right]^{-1}-\left[A s y \operatorname{Var}\left(\sqrt{T N} \hat{\boldsymbol{\beta}}_{F E}\right)\right]^{-1}=\sigma_{u}^{-2} \boldsymbol{\Omega}_{C}>0,
$$

and hence

$$
\operatorname{Asy} \operatorname{Var}\left(\sqrt{T N} \hat{\boldsymbol{\beta}}_{F E}\right)>A s y \operatorname{Var}\left(\sqrt{T N} \hat{\boldsymbol{\beta}}_{P}\right) .
$$

Consistent estimators of $\boldsymbol{\Omega}_{P}$ and $\boldsymbol{\Omega}_{F E}$ are given by $\mathbf{Q}_{N, p}$ and $\mathbf{Q}_{N, F E}$, respectively. (see $(3.14))$. 


\subsection{Random effects and PLS estimators}

Finally, it is easily seen that random effects (RE) and the pooled least squares estimators of $\boldsymbol{\beta}$ are asymptotically equivalent. The RE estimator is given by (see, for example, Chapter 26 in Pesaran (2015)).

$$
\hat{\boldsymbol{\beta}}_{R E}=\left(\mathbf{Q}_{F E, N}+\psi \mathbf{Q}_{C, N}\right)^{-1}\left(\mathbf{q}_{F E, N}+\psi \mathbf{q}_{C, N}\right),
$$

where $\mathbf{Q}_{F E, N}$ and $\mathbf{q}_{F E, N}$, are defined by (3.4),

$$
\mathbf{Q}_{C, N}=N^{-1} \sum_{i=1}^{N}\left(\overline{\mathbf{x}}_{i}-\overline{\mathbf{x}}\right)\left(\overline{\mathbf{x}}_{i}-\overline{\mathbf{x}}\right)^{\prime}, \mathbf{q}_{C, N}=N^{-1} \sum_{i=1}^{N}\left(\overline{\mathbf{x}}_{i}-\overline{\mathbf{x}}\right)\left(\bar{y}_{i}-\bar{y}\right) .
$$

and

$$
\psi=\frac{\sigma_{u}^{2}}{T \sigma_{\eta}^{2}+\sigma_{u}^{2}} .
$$

However, under $(2.5), \sigma_{\eta}^{2}=O\left(N^{\delta-1}\right)$, and for a fixed $T$, we have $\psi=1+O\left(N^{\delta-1}\right)$, and using (3.3) and (3.4) we obtain ${ }^{3}$

$$
\begin{aligned}
\mathbf{Q}_{F E, N}+\psi \mathbf{Q}_{C, N} & =(\psi-1) \mathbf{Q}_{C, N}+\mathbf{Q}_{P, N}, \\
\mathbf{q}_{F E, N}+\psi \mathbf{q}_{C, N} & =(\psi-1) \mathbf{q}_{C, N}+\mathbf{q}_{P, N}
\end{aligned}
$$

Hence (for a fixed $T$ )

$$
\sqrt{N}\left(\hat{\boldsymbol{\beta}}_{R E}-\hat{\boldsymbol{\beta}}_{P}\right) \rightarrow_{p} \mathbf{0}, \text { as } N \rightarrow \infty \text {, if } \delta<1
$$

which establishes the asymptotic equivalence of random effects and pooled least squares estimators as $N \rightarrow \infty$, for $\delta<1$ and a fixed $T$.

\section{Diagnostic test of $\delta<\frac{1}{2}$}

In the above analysis, we establish the asymptotic properties of pooled LS and FE estimator. We also compare the efficiency of PLS and FE in Proposition (3.3) and show that the PLS estimator is more efficient than the FE estimator when $\delta<\frac{1}{2}$. Hence, it would be desirable to use the PLS estimator for model (2.1) in terms of efficiency if $\delta<1 / 2$. Here we propose a Hausman type diagnostic test (Hausman, (1978)) for the test of

$$
H_{0}: \delta=\frac{1}{2}-\epsilon, \text { against } H_{1}: \delta \geq 1 / 2,
$$

\footnotetext{
${ }^{3}$ Note tha $\mathbf{Q}_{P, N}=\mathbf{Q}_{F E, N}+\mathbf{Q}_{C, N}$, and $\mathbf{q}_{P, N}=\mathbf{q}_{F E, N}+\mathbf{q}_{C, N}$.
} 
where $0<\epsilon \leq \frac{1}{2}$. Such a test will be based on the difference between the PLS and FE estimators. For PLS estimator (3.1) and FE estimator (3.2) of model (2.1), both of them are consistent, but under null hypothesis (4.1), (3.1) is more efficient than (3.2). Let

$$
\hat{\mathbf{q}}=\hat{\boldsymbol{\beta}}_{P}-\hat{\boldsymbol{\beta}}_{F E},
$$

then the Hausman test examines whether the PLS and FE estimators are significantly different. Then under the null, we have

$$
\operatorname{Var}(\hat{\mathbf{q}})=\operatorname{Var}\left(\hat{\boldsymbol{\beta}}_{F E}\right)-\operatorname{Var}\left(\hat{\boldsymbol{\beta}}_{P}\right),
$$

which can be estimated as

$$
\widehat{\operatorname{Var}}(\hat{\mathbf{q}})=\widehat{\operatorname{Var}}\left(\hat{\boldsymbol{\beta}}_{F E}\right)-\widehat{\operatorname{Var}}\left(\hat{\boldsymbol{\beta}}_{P}\right)
$$

where $\widehat{\operatorname{Var}}\left(\hat{\boldsymbol{\beta}}_{F E}\right)$ and $\widehat{\operatorname{Var}}\left(\hat{\boldsymbol{\beta}}_{P}\right)$ are the estimated covariance of $\hat{\boldsymbol{\beta}}_{F E}$ and $\hat{\boldsymbol{\beta}}_{P}$ obtained under the assumption that errors, $u_{i t}$, are serially uncorrelated and homoscedastic. Under this setting, the Hausman test statistics is given by

$$
H=\hat{\mathbf{q}}^{\prime}[\widehat{\operatorname{Var}}(\hat{\mathbf{q}})]^{-1} \hat{\mathbf{q}}
$$

which is distributed as $\chi_{k}^{2}$ for $N$ sufficiently large, where $k$ is the number of regressors in model (2.1).

If the Hausman test statistics (4.5) can't reject the null hypothesis $H_{0}$ in (4.1), then by using the result in Proposition (3.3), it is more efficient to pool the data and use the PLS estimator.

However, it should be noted that the above test does not apply if the errors $u_{i t}$ are serially correlated or cross-sectionally heteroskedastic. In this case, we can still show that both $\hat{\boldsymbol{\beta}}_{P}$ and $\hat{\boldsymbol{\beta}}_{F E}$ are consistent, but neither is efficient. Therefore, the Hausman formula for variance of the difference doesn't apply, namely $\operatorname{Var}\left(\hat{\boldsymbol{\beta}}_{P}-\hat{\boldsymbol{\beta}}_{F E}\right) \neq \operatorname{Var}\left(\hat{\boldsymbol{\beta}}_{F E}\right)-\operatorname{Var}\left(\hat{\boldsymbol{\beta}}_{P}\right)$. But we notice

$$
\hat{\mathbf{q}}=\left(\hat{\boldsymbol{\beta}}_{P}-\boldsymbol{\beta}\right)-\left(\hat{\boldsymbol{\beta}}_{F E}-\boldsymbol{\beta}\right),
$$

and

$$
E\left(\hat{\boldsymbol{\beta}}_{P}-\hat{\boldsymbol{\beta}}_{F E} \mid \mathbf{X}\right)=0
$$

by using the results in the previous section.

For the term (4.6), in order to control the effects of $\delta$ and sample sizes $(N, T)$, suppose now $T=O\left(N^{d}\right)$, for some $d>0$. In case $T$ is fixed, then $d$ is close to zero. For (4.2), we have

$$
\sqrt{N T} \hat{\mathbf{q}}=\sqrt{N T}\left(\hat{\boldsymbol{\beta}}_{P}-\boldsymbol{\beta}\right)-\sqrt{N T}\left(\hat{\boldsymbol{\beta}}_{F E}-\boldsymbol{\beta}\right),
$$


and using the results in (3.17) and (3.22) we have

$$
\sqrt{N T} \hat{\mathbf{q}}=\frac{1}{\sqrt{T N}} \sum_{i=1}^{N} \sum_{t=1}^{T}\left[\mathbf{Q}_{P, N}^{-1}\left(\mathbf{w}_{i t}-\overline{\mathbf{w}}\right)-\mathbf{Q}_{F E, N}^{-1}\left(\mathbf{w}_{i t}-\overline{\mathbf{w}}_{i}\right)\right] u_{i t}+O_{p}\left(N^{\delta+\frac{d}{2}-1 / 2}\right),
$$

where the first term will contribute to the limiting distribution, and the second will vanish if

$$
\delta+\frac{d}{2}-1 / 2<0 \text { or } \delta<\frac{1}{2}(1-d)
$$

This is the same as the poolability condition discussed in Remark 3.4

Remark 4.1 It would be very interesting to point out that both $\delta$ and $d$ have significant impact on the validity of the test. From (4.8), the second term on the RHS will disappear if and only if $\delta<\frac{1}{2}(1-d)$. By definition of $d$, we have $d=\ln T / \ln N$, thus we require $\delta<\frac{1}{2}(1-\ln T / \ln N)$. It should be noted that $\ln T / \ln N$ will not be a small number even if $T$ is fixed and $N$ is large. For instance, $d=0.1590$ if $N=1000$ and $T=3$, and $d=0.2330$ if $N=1000$ and $T=5$. As a result, the magnitude of $\ln T / \ln N$ matters for the size of the Hausman type tests, a feature which is apparent from the Monte Carlo simulations reported below.

For the implementation of the Hausman by direct derivations we have

$$
\begin{aligned}
\operatorname{Var}(\sqrt{N T} \hat{\mathbf{q}}) & =N T \times \operatorname{Var}\left(\hat{\boldsymbol{\beta}}_{P}-\hat{\boldsymbol{\beta}}_{F E}\right) \\
& \left.=\operatorname{NTV} \operatorname{Var}\left(\hat{\boldsymbol{\beta}}_{P}\right)+\operatorname{NTV} \operatorname{Var}\left(\hat{\boldsymbol{\beta}}_{F E}\right)-\operatorname{NTCov}\left(\hat{\boldsymbol{\beta}}_{F E}, \hat{\boldsymbol{\beta}}_{P}\right)-\operatorname{NTCov}\left(\hat{\boldsymbol{\beta}}_{P}, \hat{\boldsymbol{\beta}} A_{E} \phi^{\phi}\right)\right)
\end{aligned}
$$

and it can be shown that

$$
\begin{aligned}
N T \times \operatorname{Var}\left(\hat{\boldsymbol{\beta}}_{P}-\hat{\boldsymbol{\beta}}_{F E}\right)= & \mathbf{Q}_{P}^{-1} \frac{1}{N T} \sum_{i=1}^{N} \sum_{t=1}^{T} \sigma_{u}^{2} E\left[\left(\mathbf{w}_{i t}-\overline{\mathbf{w}}\right)^{2}\right] \mathbf{Q}_{P}^{-1} \\
& +\mathbf{Q}_{F E}^{-1} \frac{\sigma_{u}^{2}}{N T} \sum_{i=1}^{N} \sum_{t=1}^{T} E\left[\left(\mathbf{w}_{i t}-\overline{\mathbf{w}}_{i}\right)\left(\mathbf{w}_{i t}-\overline{\mathbf{w}}_{i}\right)^{\prime}\right] \mathbf{Q}_{F E}^{-1} \\
& -\mathbf{Q}_{F E}^{-1} \frac{\sigma_{u}^{2}}{N T} \sum_{i=1}^{N} \sum_{t=1}^{T} E\left[\left(\mathbf{w}_{i t}-\overline{\mathbf{w}}_{i}\right)\left(\mathbf{w}_{i t}-\overline{\mathbf{w}}\right)^{\prime}\right] \mathbf{Q}_{P}^{-1} \\
& \left.-\mathbf{Q}_{P}^{-1} \frac{\sigma_{u}^{2}}{N T} \sum_{i=1}^{N} \sum_{t=1}^{T} E\left[\left(\mathbf{w}_{i t}-\overline{\mathbf{w}}\right)\left(\mathbf{w}_{i t}-\overline{\mathbf{w}}_{i}\right)^{\prime}\right] \mathbf{Q}_{F E}^{-1}+O\left(N^{\delta}(4 .)\right)^{1}\right)
\end{aligned}
$$

which in turn gives

$$
\left[\sqrt{N T} \operatorname{Var}\left(\hat{\boldsymbol{\beta}}_{P}-\hat{\boldsymbol{\beta}}_{F E}\right)\right]^{-1 / 2} \sqrt{N T}\left(\hat{\boldsymbol{\beta}}_{P}-\hat{\boldsymbol{\beta}}_{F E}\right) \rightarrow_{d} N\left(0, I_{k}\right),
$$


and

$$
\left(\hat{\boldsymbol{\beta}}_{P}-\hat{\boldsymbol{\beta}}_{F E}\right)^{\prime}\left[\operatorname{Var}\left(\hat{\boldsymbol{\beta}}_{P}-\hat{\boldsymbol{\beta}}_{F E}\right)\right]^{-1}\left(\hat{\boldsymbol{\beta}}_{P}-\hat{\boldsymbol{\beta}}_{F E}\right) \rightarrow_{d} \chi_{k}^{2},
$$

i.e., the Hausman type test statistics (4.5).

For this variance term (4.11), it is infeasible since it depends on unobservable component $\mathbf{w}_{i t}$. In order to have a feasible estimator for this variance, we notice that under assumptions A1, A3-A5,

$$
\operatorname{Var}\left(\hat{\boldsymbol{\beta}}_{P}\right)=\frac{1}{N T} \mathbf{Q}_{P}^{-1} \mathbf{V}_{P} \mathbf{Q}_{P}^{-1}+o(1)
$$

where

$$
\mathbf{V}_{P}=\lim _{N \rightarrow \infty} \mathbf{V}_{P, N}=\lim _{N \rightarrow \infty} \frac{1}{N T} \sum_{i=1}^{N} \sum_{t=1}^{T} \sum_{t^{\prime}=1}^{T} \gamma_{i}\left(t, t^{\prime}\right)\left(\mathbf{w}_{i t}-\overline{\mathbf{w}}\right)\left(\mathbf{w}_{i t}-\overline{\mathbf{w}}\right)^{\prime},
$$

with $\gamma_{i}\left(t, t^{\prime}\right)=E\left(u_{i t} u_{j t^{\prime}} \mid \mathbf{X}\right)=\gamma_{i}\left(t, t^{\prime}\right)$, if $i=j$ and $t \neq t^{\prime}$. And

$$
\operatorname{Var}\left(\hat{\boldsymbol{\beta}}_{F E}\right)=\frac{1}{N T} \mathbf{Q}_{F E}^{-1} \mathbf{V}_{F E} \mathbf{Q}_{F E, N}^{-1}+o(1),
$$

where

$$
\mathbf{V}_{F E}=\lim _{N \rightarrow \infty} \mathbf{V}_{F E, N}=\lim _{N \rightarrow \infty} \frac{1}{N T} \sum_{i=1}^{N} \sum_{t=1}^{T} \sum_{t^{\prime}=1}^{T} \gamma_{i}\left(t, t^{\prime}\right)\left(\mathbf{w}_{i t}-\overline{\mathbf{w}}_{i}\right)\left(\mathbf{w}_{i t}-\overline{\mathbf{w}}_{i}\right)^{\prime} .
$$

Furthermore, we can obtain

$$
\operatorname{Cov}\left(\hat{\boldsymbol{\beta}}_{F E}, \hat{\boldsymbol{\beta}}_{P}\right)=\frac{1}{N T} \mathbf{Q}_{F E}^{-1} \mathbf{V}_{F E P} \mathbf{Q}_{P}^{-1}+o(1),
$$

where

$$
\mathbf{V}_{F E P}=\lim _{N \rightarrow \infty} \mathbf{V}_{F E P, N}=\lim _{N \rightarrow \infty} \frac{1}{N T} \sum_{i=1}^{N} \sum_{t=1}^{T} \sum_{t^{\prime}=1}^{T} \gamma_{i}\left(t, t^{\prime}\right)\left(\mathbf{w}_{i t}-\overline{\mathbf{w}}_{i}\right)\left(\mathbf{w}_{i t^{\prime}}-\overline{\mathbf{w}}\right)^{\prime},
$$

and

$$
\operatorname{Cov}\left(\hat{\boldsymbol{\beta}}_{P}, \hat{\boldsymbol{\beta}}_{F E}\right)=\frac{1}{N T} \mathbf{Q}_{P}^{-1} \mathbf{V}_{P F E} \mathbf{Q}_{F E}^{-1}+o(1),
$$

with

$$
\mathbf{V}_{P F E}=\lim _{N \rightarrow \infty} \mathbf{V}_{P F E, N}=\lim _{N \rightarrow \infty} \frac{1}{N T} \sum_{i=1}^{N} \sum_{t=1}^{T} \sum_{t^{\prime}=1}^{T} \gamma_{i}\left(t, t^{\prime}\right)\left(\mathbf{w}_{i t^{\prime}}-\overline{\mathbf{w}}\right)\left(\mathbf{w}_{i t}-\overline{\mathbf{w}}_{i}\right)^{\prime}
$$

Hence combining (4.12)-(4.18) yields

$$
\operatorname{Var}\left(\hat{\boldsymbol{\beta}}_{P}-\hat{\boldsymbol{\beta}}_{F E}\right)=\frac{1}{N T}\left[\begin{array}{c}
\mathbf{Q}_{F E}^{-1} \mathbf{V}_{F E} \mathbf{Q}_{F E}^{-1}+\mathbf{Q}_{P}^{-1} \mathbf{V}_{P} \mathbf{Q}_{P}^{-1} \\
-\mathbf{Q}_{F E}^{-1} \mathbf{V}_{F E P} \mathbf{Q}_{P}^{-1}-\mathbf{Q}_{P}^{-1} \mathbf{V}_{P F E} \mathbf{Q}_{F E}^{-1}
\end{array}\right]
$$


which reduces to the standard formula if it is assumed that the errors are serially uncorrelated and homoskedastic. To see this note that in the case of serially uncorrelated errors, $\gamma_{i}\left(t, t^{\prime}\right)=0$ if $t \neq t^{\prime}$, and $\gamma_{i}(t, t)=\sigma_{u}^{2}$, we have

$$
\begin{aligned}
\mathbf{V}_{F E P} & =\lim _{N \rightarrow \infty} \frac{1}{N T} \sum_{i=1}^{N} \sum_{t=1}^{T} \sigma_{u}^{2}\left(\mathbf{w}_{i t}-\overline{\mathbf{w}}_{i}\right)\left(\mathbf{w}_{i t}-\overline{\mathbf{w}}\right)^{\prime} \\
& =\lim _{N \rightarrow \infty} \frac{1}{N T} \sum_{i=1}^{N} \sum_{t=1}^{T} \sigma_{u}^{2}\left(\mathbf{w}_{i t}-\overline{\mathbf{w}}_{i}\right)\left[\left(\mathbf{w}_{i t}-\overline{\mathbf{w}}_{i}\right)+\left(\overline{\mathbf{w}}_{i}-\overline{\mathbf{w}}\right)\right]^{\prime} \\
& =\sigma_{u}^{2} \boldsymbol{\Omega}_{F E}+\lim _{N \rightarrow \infty} \frac{1}{N T} \sum_{i=1}^{N} \sum_{t=1}^{T} \sigma_{u}^{2}\left(\mathbf{w}_{i t}-\overline{\mathbf{w}}_{i}\right)\left(\overline{\mathbf{w}}_{i}-\overline{\mathbf{w}}\right)^{\prime} \\
& =\sigma_{u}^{2} \boldsymbol{\Omega}_{F E} .
\end{aligned}
$$

Similarly, $\mathbf{V}_{P F E}=\sigma_{u}^{2} \boldsymbol{\Omega}_{F E}$. Therefore, in this case

$$
\begin{aligned}
\operatorname{Var}\left(\hat{\boldsymbol{\beta}}_{P}-\hat{\boldsymbol{\beta}}_{F E} \mid \mathbf{X}\right) & =\frac{1}{N T} \sigma_{u}^{2}\left[\begin{array}{c}
\boldsymbol{\Omega}_{F E}^{-1} \boldsymbol{\Omega}_{F E} \boldsymbol{\Omega}_{F E}^{-1}+\boldsymbol{\Omega}_{P}^{-1} \boldsymbol{\Omega}_{P} \boldsymbol{\Omega}_{P}^{-1} \\
-\boldsymbol{\Omega}_{F E}^{-1} \boldsymbol{\Omega}_{F E} \boldsymbol{\Omega}_{P}^{-1}-\boldsymbol{\Omega}_{P}^{-1} \boldsymbol{\Omega}_{F E} \boldsymbol{\Omega}_{F E}^{-1}
\end{array}\right] \\
& =\frac{1}{N T} \sigma_{u}^{2}\left(\boldsymbol{\Omega}_{F E}^{-1}-\boldsymbol{\Omega}_{P}^{-1}\right) \\
& =\operatorname{Var}\left(\hat{\boldsymbol{\beta}}_{F E}\right)-\operatorname{Var}\left(\hat{\boldsymbol{\beta}}_{P}\right)
\end{aligned}
$$

which accords with the Hausman's variance formula in (4.3).

Given the consistent estimator of (4.20), a general Hausman test statistics of (4.1) has the form

$$
\tilde{H}=\hat{\mathbf{q}}^{\prime}\left[\operatorname{Var}\left(\widehat{\hat{\boldsymbol{\beta}}_{P}-} \hat{\boldsymbol{\beta}}_{F E}\right)\right]^{-1} \hat{\mathbf{q}}
$$

which is distributed as $\chi_{k}^{2}$ for $N$ sufficiently large. In the general case where the errors are serially correlated or cross-sectionally heteroskedastic, using (4.20), under the null hypothesis, $\operatorname{Var}\left(\hat{\boldsymbol{\beta}}_{P}-\hat{\boldsymbol{\beta}}_{F E}\right)$ can be consistently estimated by ${ }^{4}$

$$
\operatorname{Var}\left(\widehat{\hat{\boldsymbol{\beta}}_{P}-} \hat{\boldsymbol{\beta}}_{F E}\right)=\frac{1}{N T}\left[\begin{array}{c}
\mathbf{Q}_{F E, N}^{-1} \hat{\mathbf{V}}_{F E, N} \mathbf{Q}_{F E, N}^{-1}+\mathbf{Q}_{P, N}^{-1} \hat{\mathbf{V}}_{P, N T} \mathbf{Q}_{P, N}^{-1} \\
-\mathbf{Q}_{F E, N}^{-1} \hat{\mathbf{V}}_{F E P, N} \mathbf{Q}_{P, N}^{-1}-\mathbf{Q}_{P, N}^{-1} \hat{\mathbf{V}}_{P F E, N} \mathbf{Q}_{F E, N}^{-1}
\end{array}\right],
$$

where (see (3.14) and (3.20))

$$
\mathbf{Q}_{F E, N}=\frac{1}{N T} \sum_{i=1}^{N} \sum_{t=1}^{T}\left(\mathbf{x}_{i t}-\overline{\mathbf{x}}_{i}\right)\left(\mathbf{x}_{i t}-\overline{\mathbf{x}}_{i}\right)^{\prime} ; \mathbf{Q}_{P, N}=\frac{1}{N T} \sum_{i=1}^{N} \sum_{t=1}^{T}\left(\mathbf{x}_{i t}-\overline{\mathbf{x}}\right)\left(\mathbf{x}_{i t}-\overline{\mathbf{x}}\right)^{\prime}
$$

\footnotetext{
${ }^{4}$ See Pesaran (2015, pp 653-655).
} 
and

$$
\begin{aligned}
\hat{\mathbf{V}}_{F E, N} & =\frac{1}{N T} \sum_{i=1}^{N} \mathbf{X}_{i}^{\prime} \mathbf{M}_{T} \hat{\mathbf{u}}_{i}^{*} \hat{\mathbf{u}}_{i}^{* \prime} \mathbf{M}_{T} \mathbf{X}_{i}, \hat{\mathbf{V}}_{P, N T}=\frac{1}{N T} \sum_{i=1}^{N} \tilde{\mathbf{X}}_{i}^{\prime} \hat{\mathbf{u}}_{i, O L S} \hat{\mathbf{u}}_{i, O L S}^{\prime} \tilde{\mathbf{X}}_{i}, \\
\hat{\mathbf{V}}_{F E P, N} & =\frac{1}{N T} \sum_{i=1}^{N} \mathbf{X}_{i}^{\prime} \mathbf{M}_{T} \hat{\mathbf{u}}_{i, O L S} \hat{\mathbf{u}}_{i, O L S}^{\prime} \tilde{\mathbf{X}}_{i}, \hat{\mathbf{V}}_{P F E, N}=\hat{\mathbf{V}}_{F E P, N}^{\prime},
\end{aligned}
$$

where $\mathbf{X}_{i}^{\prime}=\left(\mathbf{x}_{i 1}, \mathbf{x}_{i 2}, \ldots, \mathbf{x}_{i T}\right), \hat{\mathbf{u}}_{i}^{*}=\mathbf{M}_{T}\left(\mathbf{y}_{i}-\mathbf{X}_{i} \hat{\boldsymbol{\beta}}_{F E}\right), \tilde{\mathbf{X}}_{i}^{\prime}=\left(\mathbf{x}_{i 1}-\overline{\mathbf{x}}, \mathbf{x}_{i 2}-\overline{\mathbf{x}}, \ldots, \mathbf{x}_{i T}-\overline{\mathbf{x}}\right)$, $\hat{\mathbf{u}}_{i, O L S}=\left(\hat{u}_{i 1, O L S}, \hat{u}_{i 2, O L S}, \ldots, \hat{u}_{i T, O L S}\right)^{\prime}$ with $\hat{u}_{i t, O L S}=y_{i t}-\bar{y}-\left(\mathbf{x}_{i t}-\overline{\mathbf{x}}\right)^{\prime} \hat{\boldsymbol{\beta}}_{P}$ for $t=1, \ldots, T$, and $\mathbf{M}_{T}=\mathbf{I}_{T}-\boldsymbol{\tau}_{T}\left(\boldsymbol{\tau}_{T}^{\prime} \boldsymbol{\tau}_{T}\right)^{-1} \boldsymbol{\tau}_{T}^{\prime}$ with $\boldsymbol{\tau}_{T}$ being a vector of ones of length $T$.

\section{Monte Carlo simulations}

To compare the performance of the FE and pooled least square estimators when $T$ is fixed as well as $\sum_{i=1}^{N}\left|\eta_{i}\right|=O\left(N^{\delta}\right)$, we conduct several Monte Carlo simulations. The data generating process (DGP) is given by

$$
y_{i t}=1+\eta_{i}+x_{1, i t} \beta_{1}+x_{2, i t} \beta_{2}+u_{i t}, \quad i=1,2, \ldots, N ; t=1,2, \ldots, T,
$$

with $\beta_{1}=1$ and $\beta_{2}=2, N=100,500,1000,2000$ and $T=3,5,10$. We assume $u_{i t} \sim i i d N\left(0, \sigma_{i}^{2}\right)$, with $\sigma_{i}^{2} \sim I I D \chi^{2}(2), \eta_{i} \sim \operatorname{iidN}(0,2)$ for $i=1,2, \ldots,\left[N^{\delta}\right]$ and $\eta_{i}=0$, for $i=\left[N^{\delta}\right]+1,\left[N^{\delta}\right]+$ $2, \ldots, N$. We let $\delta$ to take the following values $1,0.95,0.75,0.5,0.4,0.25$ and 0 . The elements of $\mathbf{x}_{i t}=\left(x_{1, i t}, x_{2, i t}\right)^{\prime}$, are generated as

$$
x_{j, i t}=1+\alpha_{j, i}+g_{j, t} \eta_{i}+w_{j, i t}, \text { for } j=1,2,
$$

with $\alpha_{j, i} \sim \operatorname{iidN}(0,1), g_{j, t} \sim \operatorname{IIDU}[0.1,0.9]$ and $w_{j, i t}$ generated by

$$
w_{j, i t}=\rho_{j, i} w_{k, i t-1}+\varepsilon_{j, i t}, \text { for } j=1,2,
$$

where $w_{j, i 0}=0, \rho_{j, i} \sim \operatorname{IIDU}[0.05,0.95], \varepsilon_{j, i 0}=0$, and $\varepsilon_{j, i t} \sim \operatorname{iidN}\left(0, \sigma_{j, \varepsilon i}^{2}\right)$ with $\sigma_{j, \varepsilon i}^{2} \sim$ $I I D \chi^{2}(2)$ for $j=1,2$. For the DGP described above, the first 50 observations are discarded, and the number of replications is set to 1000 .

We compute the PLS and FE estimates and the associated bias, absolute bias and RMSE. These estimation results are summarized in Table 1-6. As suggested by the theory the RMSE of the PLS estimator is much smaller than those of the FE estimator for values of $\delta<1 / 2$. However, the PLS estimator starts to show significant bias as $\delta$ is allowed to increase beyond the $1 / 2$ threshold, and the RMSE of PLS estimator is much larger than the FE estimator. 
The results of the poolability test using the Hausman type statistic are summarized in Table 7. We can observe that the empirical size is very close to the $5 \%$ nominal value when $\delta<0.25$, which makes sense since for all our combinations of $(N, T)$, the minimum $d$ is 0.1445 and the maximum $d$ is 0.5 . The proposed poolability test has good power for values of $\delta>(1-d) / 2$, as predicted by the theory.

\section{Conclusion}

This paper introduces a new approach to the analysis of the relative efficiency of fixed effects and pooled least square estimators for standard panel data models. We show that the potential benefit from pooling is directly related to the degree with which the heterogeneity of individual effects is pervasive across the individual units in the panel. We characterize this feature by an exponent, $\delta$, and show that pooled least square estimator is consistent for values of $\delta<1$. Our specification allows for non-zero correlations between the individual effects and the regressors which renders the pooled least squares and random effects inconsistent if $\delta=1$. We also derive the asymptotic distributions of the pooled least squares, FE and RE estimators for different values of $\delta$ and establish the relative efficiency of the pooled least squares estimator over the FE estimator when $\delta<(1-d) / 2$, where $d$ is given by $\ln T / \ln N$. We also propose a Hausman type

diagnostic test of poolability. The theoretical results are supported by small sample evidence from Monte Carlo experiments.

\section{References}

[1] Bailey, N., G. Kapetanios, and M.H. Pesaran, 2016, Exponent of cross-sectional dependence: estimation and inference, Journal of Applied Econometrics 31, 929-960.

[2] Baltagi, B., G. Bresson and A. Pirotte, 2008, To Pool or Not to Pool?, Chapter 16 in László Mátyás, Patrick Sevestre (eds.), The Econometrics of Panel Data, 517-546, Springer Berlin Heidelberg;

[3] Baltagi, B., J. Griffin, and W. Xiong, 2000, To pool or not to pool: homogeneous versus hetergeneous estimations applied to cigarette demand, The Review of Economics and Statistics $82,117-126$.

[4] Hausman, J. A. 1978. Specification tests in econometrics. Econometrica 46, 1251-1272.

[5] Mundlak, Y. 1978. On the pooling of time series and cross section data. Econometrica 46, 69-85. 
[6] Paap, R, W. Wang, and X. Zhang 2015. To pool or not to pool: What is a good strategy?, manuscript.

[7] Pesaran, M. H., 2015, Time Series and Panel Data Econometrics, Oxford University Press, Oxford. 


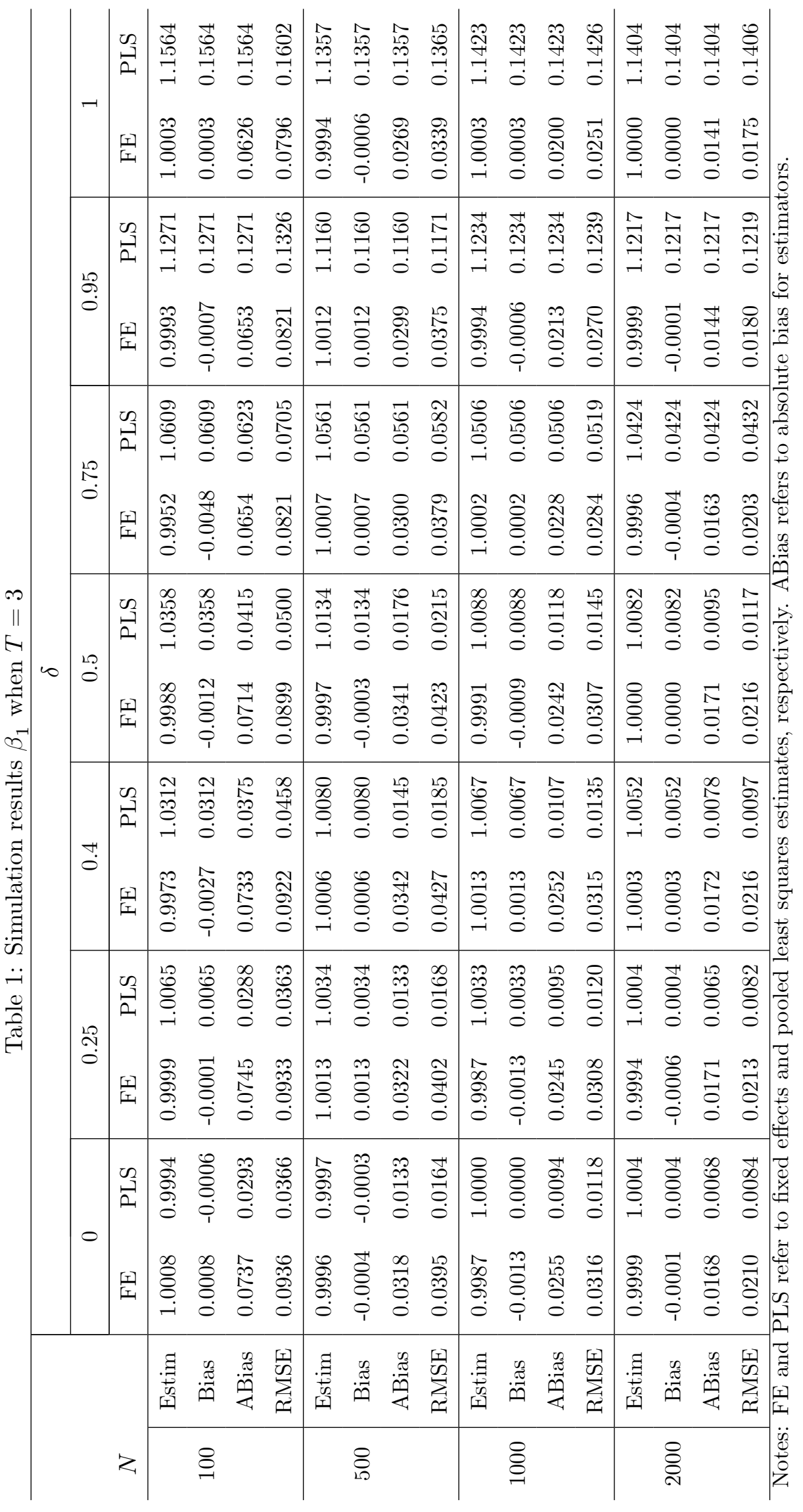




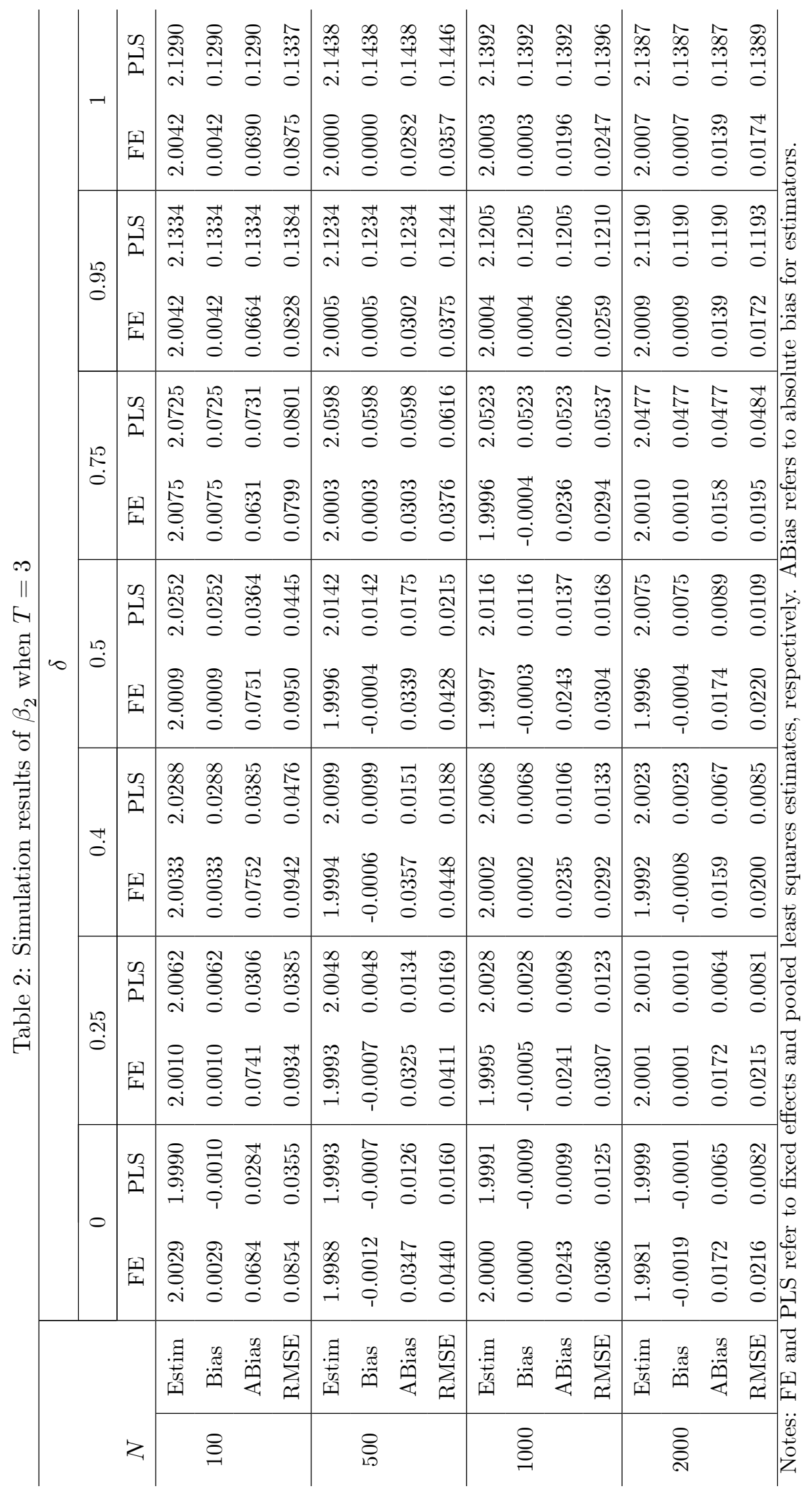




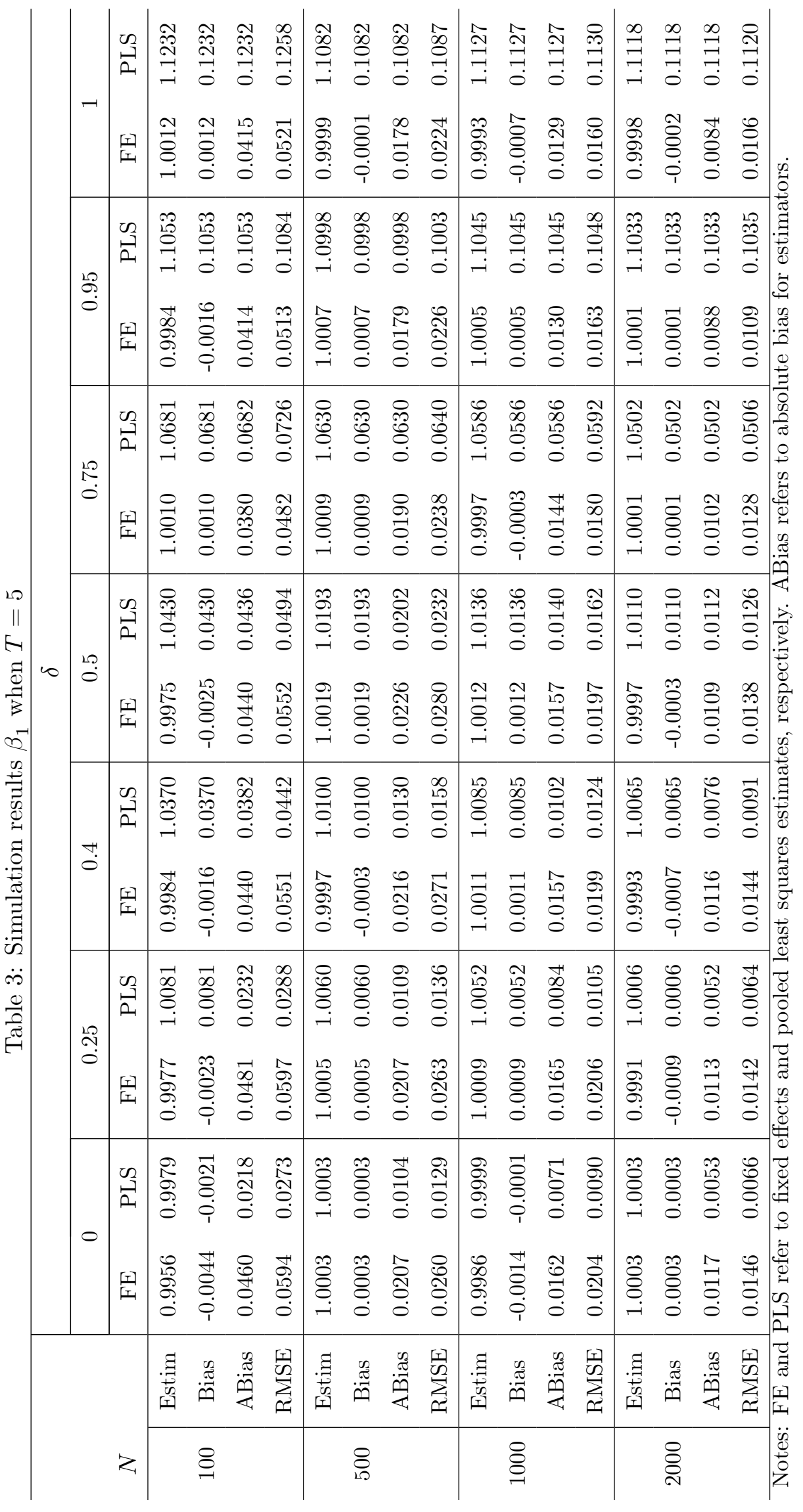




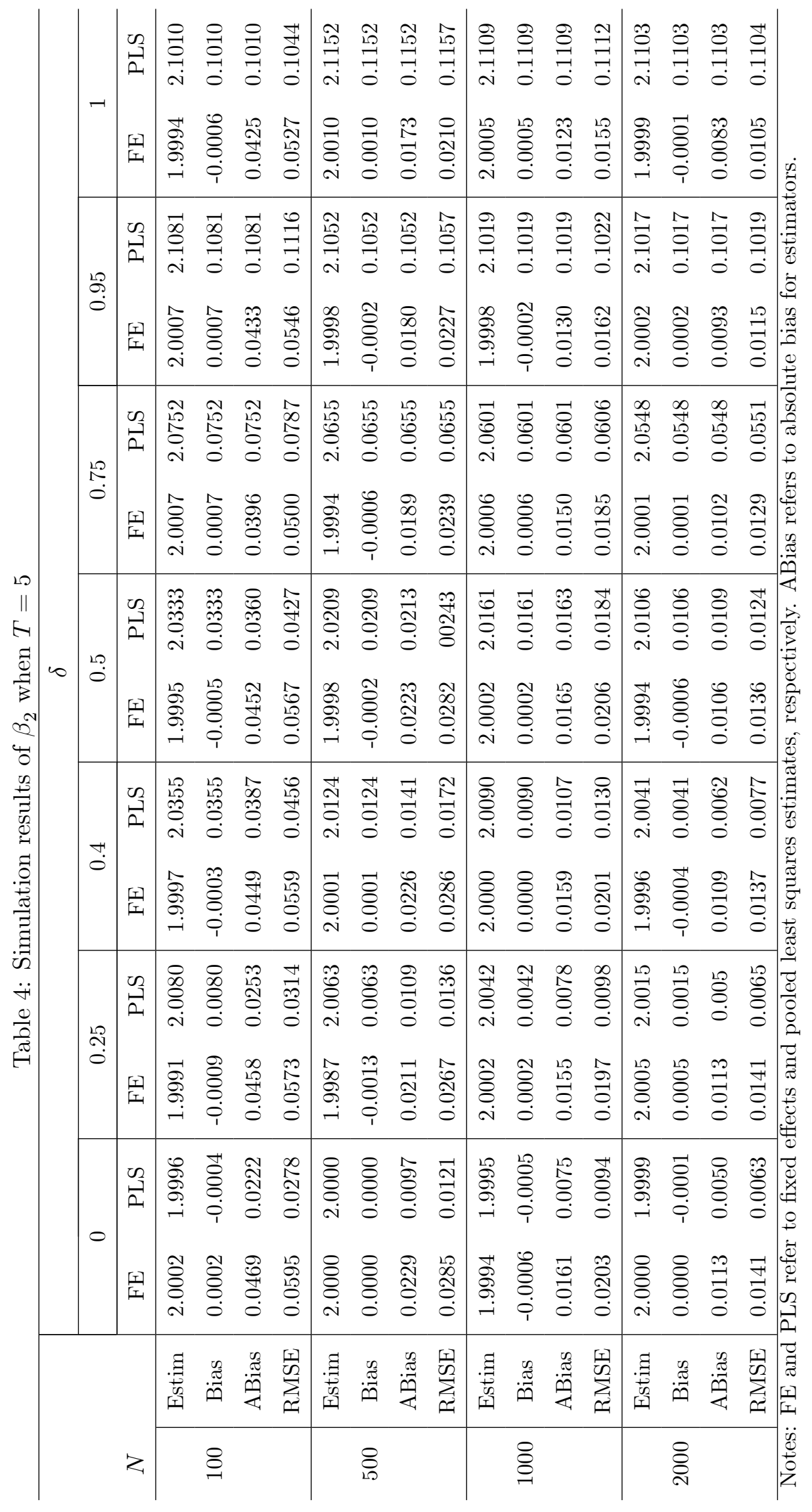




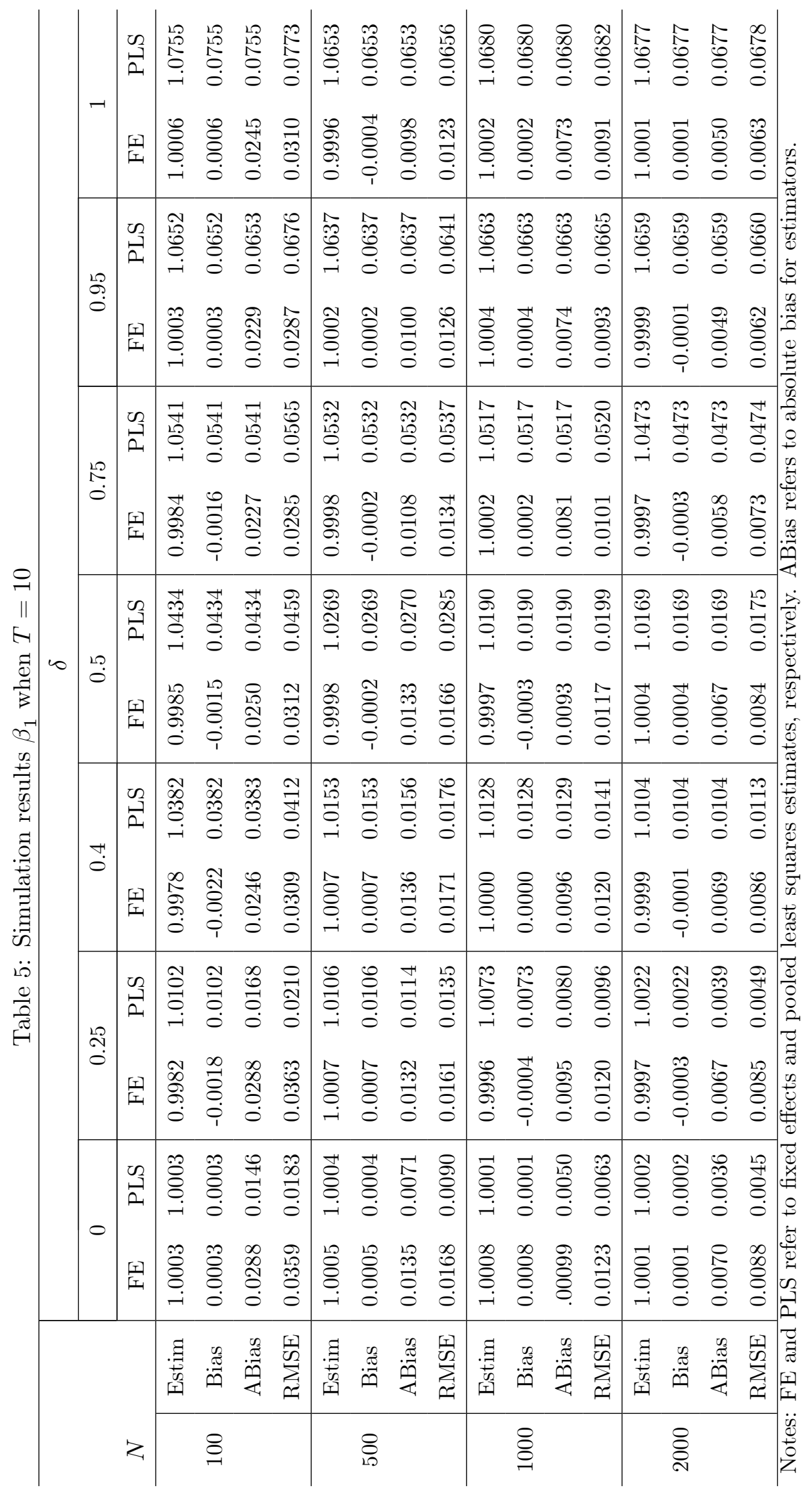




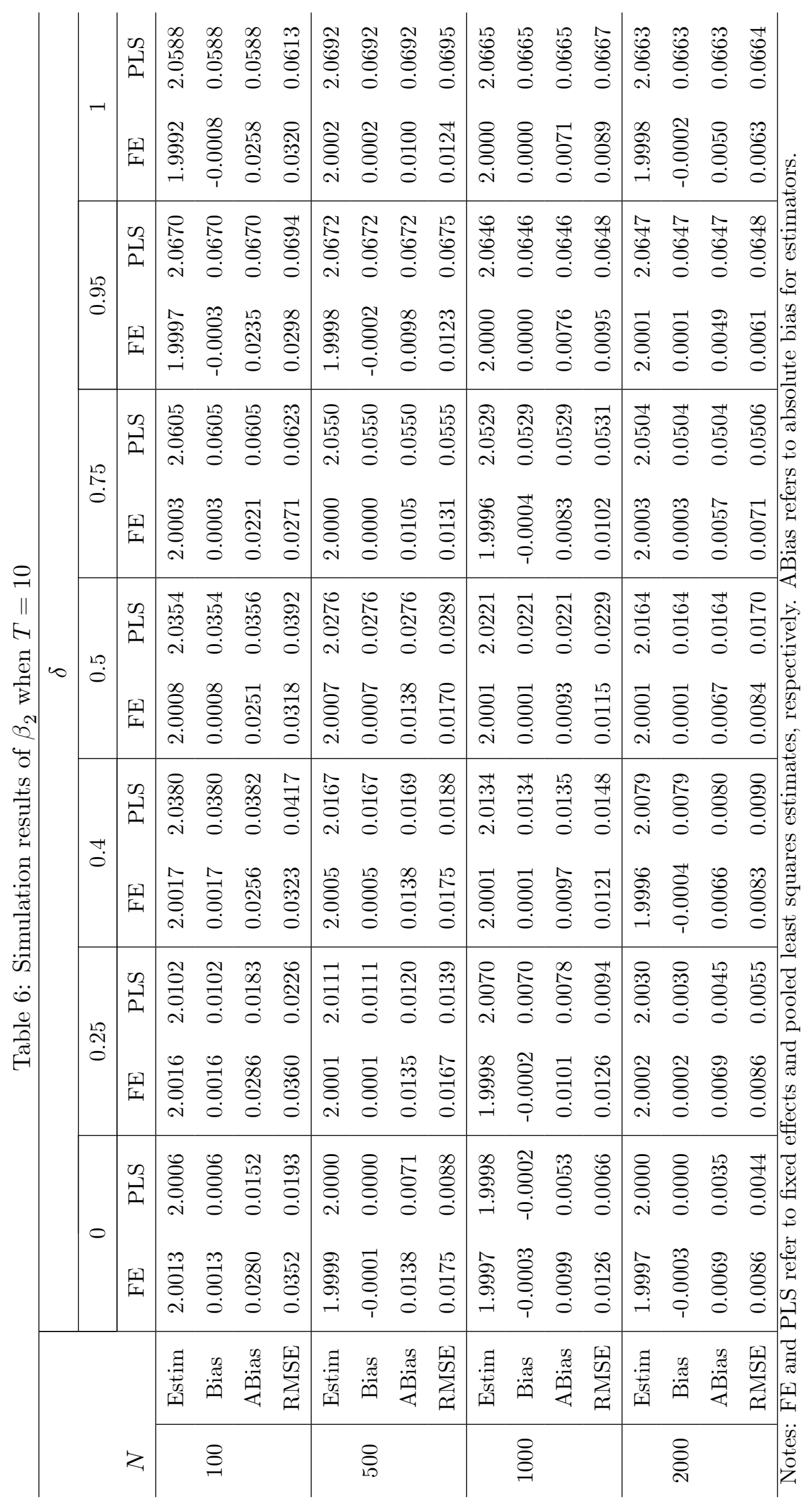


Table 7: Empirical rejection frequencies for the Hausman type test at 5\% significance level

\begin{tabular}{cc|c|c|c|c|c|c|c|c|c}
\hline & & \multicolumn{10}{c}{$\delta$} \\
\cline { 3 - 10 }$N$ & $T$ & 0 & 0.25 & 0.4 & 0.45 & 0.5 & 0.55 & 0.75 & 0.95 & 1 \\
\hline \multirow{3}{*}{100} & 3 & $6.1 \%$ & $7.4 \%$ & $7.6 \%$ & $7.3 \%$ & $8.7 \%$ & $8.8 \%$ & $22.5 \%$ & $74.2 \%$ & $90.6 \%$ \\
& 5 & $7.6 \%$ & $5.7 \%$ & $8.2 \%$ & $8.1 \%$ & $10.2 \%$ & $15.5 \%$ & $56.7 \%$ & $99.4 \%$ & $100 \%$ \\
& 10 & $5.2 \%$ & $6.7 \%$ & $14.2 \%$ & $12.2 \%$ & $20.7 \%$ & $3.85 \%$ & $98.5 \%$ & $100 \%$ & $100 \%$ \\
\hline \multirow{3}{*}{500} & 3 & $5.6 \%$ & $5.2 \%$ & $7.5 \%$ & $8.2 \%$ & $8.5 \%$ & $11.5 \%$ & $59.8 \%$ & $100 \%$ & $100 \%$ \\
& 5 & $5.9 \%$ & $7 \%$ & $7.4 \%$ & $14.4 \%$ & $13.2 \%$ & $26.8 \%$ & $99.2 \%$ & $100 \%$ & $100 \%$ \\
& 10 & $4.6 \%$ & $5.6 \%$ & $11.3 \%$ & $37.9 \%$ & $36.9 \%$ & $88.5 \%$ & $100 \%$ & $100 \%$ & $100 \%$ \\
\hline & 3 & $7 \%$ & $6 \%$ & $6 \%$ & $7 \%$ & $8.1 \%$ & $9.2 \%$ & $71.3 \%$ & $100 \%$ & $100 \%$ \\
& 5 & $7.1 \%$ & $5.9 \%$ & $8.1 \%$ & $13.4 \%$ & $14.5 \%$ & $26.8 \%$ & $100 \%$ & $100 \%$ & $100 \%$ \\
& 10 & $5.2 \%$ & $6.2 \%$ & $21.9 \%$ & $23 \%$ & $53.9 \%$ & $94.4 \%$ & $100 \%$ & $100 \%$ & $100 \%$ \\
\hline & 3 & $4.2 \%$ & $5.4 \%$ & $5.9 \%$ & $8.3 \%$ & $9.2 \%$ & $10 \%$ & $88.7 \%$ & $100 \%$ & $100 \%$ \\
& 5 & $4.5 \%$ & $6 \%$ & $6.9 \%$ & $15.4 \%$ & $18.4 \%$ & $31.5 \%$ & $100 \%$ & $100 \%$ & $100 \%$ \\
& 10 & $5.2 \%$ & $4.7 \%$ & $20.2 \%$ & $51.4 \%$ & $71.7 \%$ & $97 \%$ & $100 \%$ & $100 \%$ & $100 \%$ \\
\hline
\end{tabular}

Note: The $5 \%$ significance level for $\chi^{2}(2)$ is 5.991 . 